\title{
THE GLOBAL EXISTENCE PROBLEM IN GENERAL RELATIVITY
}

\author{
LARS ANDERSSON ${ }^{1}$
}

\begin{abstract}
We survey some known facts and open questions concerning the global properties of $3+1$ dimensional spacetimes containing a compact Cauchy surface. We consider spacetimes with an $\ell$-dimensional Lie algebra of space-like Killing fields. For each $\ell \leq 3$, we give some basic results and conjectures on global existence and cosmic censorship.
\end{abstract}

\section{COntents}

\begin{tabular}{|lr|}
\hline 1. & Introduction \\
\hline $2 . \quad$ The Einstein equations & 1 \\
\hline $3 . \quad$ Bianchi & 3 \\
\hline $4 . \quad G_{2}$ & 15 \\
\hline $5 . \quad \mathrm{U}(1)$ \\
\hline $6 . \quad 3+1$ & 23 \\
\hline $7 . \quad$ Concluding remarks & 30 \\
\hline Appendix A. Basic Causality concepts & 34 \\
\hline References & 39 \\
\hline
\end{tabular}

\section{INTRODUCTION}

In this review, we will describe some results and conjectures about the global structure of solutions to the Einstein equations in $3+1$ dimensions. We consider spacetimes $(\bar{M}, \bar{g})$ with an $\ell$-dimensional Lie algebra of spacelike Killing fields. We may say that such spacetimes have a (local) isometry group $G$ of dimension $\ell$ with the action of $G$ generated by space-like Killing fields.

For each value $\ell \leq 3$ of the dimension of the isometry group, we state the reduced field equations as well as attempt to give an overview of the most important results and conjectures. We will concentrate on the vacuum case.

In section 2] we present the Einstein equations and give the $3+1$ decomposition into constraint and evolution equations, cf. subsection 2.2 Due to

Date: June 27, 2004.

${ }^{1}$ Supported in part by the Swedish Natural Sciences Research Council, contract no. F-FU 4873-307, and the NSF under contract no. DMS 0104402. 
the gauge freedom in the Einstein equations, questions on the global properties of solutions to the Einstein equations must be posed carefully. We introduce the notions of vacuum extension and maximal Cauchy development and state the uniqueness theorem of Choquet-Bruhat and Geroch, for maximal vacuum Cauchy developments. We also collect here a few basic facts about Killing fields on globally hyperbolic spacetimes. In subsection 2.1 a version of the cosmic censorship conjecture appropriate for vacuum spacetimes, with compact Cauchy surface, is stated and in subsection 2.3 we discuss a few gauge conditions which may be of use for the global existence problem for the Einstein equations. Section 2 is ended by a discussion of a few PDE aspects of the Einstein equations which are relevant for the topic of this paper, cf. subsection 2.4

In the cases $\ell=3$ (Bianchi, cf. section 13) and a special case of $\ell=$ 2 (polarized Gowdy, cf. section 4), the global behavior of the Einstein equations is well understood, both with regard to global existence of the evolution equations and the cosmic censorship problem. For the general $\ell=2$ case (local $\mathrm{U}(1) \times \mathrm{U}(1)$ symmetry, cf. section 4 ), there are only partial results on the global existence problem and the cosmic censorship problem remains open, although conjectures supported by numerical evidence give a good indication of what the correct picture is.

In the cases $\ell=1$ (U(1) symmetry, cf. section 5) and $\ell=0$ (no symmetry, i.e. full $3+1$ dimensional Einstein equations, cf. section [6), the large data global existence and cosmic censorship problems are open. In the U(1) case conjectures supported by numerical evidence give a good idea of the generic behavior, and there is a small data semi-global existence result for the expanding direction due to Choquet-Bruhat and Moncrief [42, 39].

For $3+1$ Einstein gravity without symmetries the only global existence results known are the theorem on nonlinear stability of Minkowski space of Christodoulou and Klainerman, the semi-global existence theorem of Friedrich for the hyperboloidal initial value proble and the semi-global existence theorem for spatially compact spacetimes with Cauchy surface of hyperbolic type, due to Andersson and Moncrief. These are all small data results, see section 6 for discussion.

Due to the high degree of complexity of the numerical solution of the Einstein equations in $3+1$ dimensions it is too early to draw any conclusions relevant to the asymptotic behavior at the singularity for the full $3+1$ dimensional Einstein equations, from the numerical studies being performed. However, an attractive scenario is given by the so called BKL picture, cf. section 7 for some remarks and references.

The Einstein equations are derived from a variational principle, and can be formulated as a Hamiltonian system (or time-dependent Hamiltonian system, depending on the gauge), and therefore the Hamiltonian aspect of the dynamics should not be ignored, see eg. the work by Fischer and Moncrief on the Hamiltonian reduction of the Einstein equations, 67 and references therein. In fact, the Hamiltonian point of view on the Einstein equations has 
played a vital role as motivation and guide in the development of the results discussed here. The notion that the Einstein evolution equations in terms of canonical variables can be viewed as the geodesic spray for a metric on the phase space (deWitt metric) modified by a curvature potential, is natural from the Hamiltonian point of view, and this picture has been relevant to the development of ideas on asymptotic velocity dominance, see sections 4 and 5 .

In this review, however, we will concentrate exclusively on the differential geometric and analytical point of view. Even with this restriction, many important topics are left out and we make no claim of complete coverage. See also [139] and [110] for related surveys.

Acknowledgements: I am grateful to Vince Moncrief for numerous conversations on the topics covered here and for detailed comments on an early version. Thanks are due to Håkan Andréasson, Piotr Chruściel, Jim Isenberg and Alan Rendall and others for helpful comments. I am happy to acknowledge the hospitality and support of the Institute of Theoretical Physics, UCSB, and Institut des Hautes Études Scientifiques, Bures sur Yvette, where where part of the writing was done.

\section{The Einstein Equations}

Let $(\bar{M}, \bar{g})$ be a smooth 4-dimensional Lorentz manifold ${ }^{1}$ of signature -+++ . The Lorentzian metric $\bar{g}$ defines a causal structure on $\bar{M}$. For the convenience of the reader we give a quick review of the basic causality concepts in appendix [A] see [165, Chapter 8], 24, 84, 132] for details.

We will here consider only the vacuum case, i.e. the case when $\bar{g}$ is Ricci flat,

$$
\bar{R}_{a b}=0 \text {. }
$$

Let $M \subset \bar{M}$ be a space-like hypersurface, i.e. a hypersurface with time-like normal $T$. We let $e_{i}$ be a frame on $M$ and use indices $i, j, k$ for the frame components. Let $g, k$ be the induced metric and second fundamental form of $M \subset \bar{M}$, where $k_{i j}=\left\langle\bar{\nabla}_{i} e_{j}, T\right\rangle$. Let $t$ be a time function on a neighborhood of $M$. Then we can introduce local coordinates $\left(t, x^{i}, i=1,2,3\right)$ on $\bar{M}$ so that $x^{i}$ are coordinates on the level sets $M_{t}$ of $t$. This defines the coordinate vector field $\partial_{t}$ of $t$. Alternatively we can let $M_{t}=\mathbf{i}(t, M)$ where $\mathbf{i}: \mathbb{R} \times M \rightarrow$ $\bar{M}$ is a 1-parameter family of imbeddings of an abstract 3-manifold $M$. Then $\partial_{t}=\mathbf{i}_{*} d / d t$ where $d / d t$ is the coordinate derivative on $\mathbb{R}$.

Define the lapse $N$ and shift $X$ w.r.t. $t$ by $\partial_{t}=N T+X$. Assume that $T$ is future oriented so that $N>0$. A $3+1$ split of equation (2.1) gives the

\footnotetext{
${ }^{1}$ We denote the covariant derivative and curvature tensors associated to $(\bar{M}, \bar{g})$ by $\bar{\nabla}, \bar{R}_{a b c d}$ etc. All manifolds are assumed to be Hausdorff, second countable and $C^{\infty}$, and all fields are assumed to be $C^{\infty}$ unless otherwise stated.
} 
Einstein vacuum constraint equations

$$
\begin{aligned}
R-|k|^{2}+(\operatorname{tr} k)^{2} & =0, \\
\nabla_{i} \operatorname{tr} k-\nabla^{j} k_{i j} & =0,
\end{aligned}
$$

and the Einstein vacuum evolution equations

$$
\begin{aligned}
& \mathcal{L}_{\partial_{t}} g_{i j}=-2 N k_{i j}+\mathcal{L}_{X} g_{i j}, \\
& \mathcal{L}_{\partial_{t}} k_{i j}=-\nabla_{i} \nabla_{j} N+N\left(R_{i j}+\operatorname{tr} k k_{i j}-2 k_{i m} k_{j}^{m}\right)+\mathcal{L}_{X} k_{i j} .
\end{aligned}
$$

where $\mathcal{L}_{\partial_{t}}$ denotes Lie-derivative ${ }^{2}$ w.r.t. $\partial_{t}$. In case $\left[\partial_{t}, e_{i}\right]=0, \mathcal{L}_{\partial_{t}}$ can be replaced by $\partial_{t}$.

A triple $(M, g, k)$ consisting of a $3-$ manifold $M$, a Riemannian metric $g$ on $M$ and a symmetric covariant 2 -tensor $k$ is a vacuum data set for the Einstein equations if it solves (2.2).

Definition 2.1. Let $(M, g, k)$ be a vacuum data set.

(1) A vacuum spacetime $(\bar{M}, \bar{g})$ is called a vacuum extension of $(M, g, k)$ if there there is an imbedding $\mathbf{i}$ with time-like normal $T$ of $(M, g, k)$ into $(\bar{M}, \bar{g})$ so that $g=\mathbf{i}^{*} \bar{g}$ and $k=-\mathbf{i}^{*}(\bar{\nabla} T)$.

(2) A globally hyperbolic vacuum spacetime $(\bar{M}, \bar{g})$ is called a vacuum Cauchy development of $(M, g, k)$ if there is an imbedding $\mathbf{i}$ with time-like normal $T$ of $(M, g, k)$ into $(\bar{M}, \bar{g})$ so that $\mathbf{i}(M)$ is a Cauchy surface in $(\bar{M}, \bar{g}), g=\mathbf{i}^{*} \bar{g}$ and $k=-\mathbf{i}^{*}(\bar{\nabla} T)$. If $(\bar{M}, \bar{g})$ is maximal in the class of vacuum Cauchy developments of $(M, g, k)$ then $(\bar{M}, \bar{g})$ is called the maximal vacuum Cauchy development (MVCD) of $(M, g, k)$. In the following, when convenient, we will identify $M$ with $\mathbf{i}(M)$.

The Einstein vacuum equations are not hyperbolic in any standard sense due to the coordinate invariance ("general covariance") of the equation $\bar{R}_{a b}=0$. Nevertheless, the Cauchy problem for the Einstein vacuum equation is well posed in the following sense.

Theorem 2.2 (Choquet-Bruhat and Geroch [40). Let $(M, g, k)$ be a vacuum data set. Then there is a unique, up to isometry, maximal vacuum Cauchy development (MVCD) of $(M, g, k)$. If $\phi: M \rightarrow M$ is a diffeomorphism, the MVCD of $\left(M, \phi^{*} g, \phi^{*} k\right)$ is isometric to the MVCD of $(M, g, k)$.

The proof relies on the fact that in spacetime harmonic coordinates, $\square_{\bar{g}} x^{\alpha}=0$, the Ricci tensor is of the form

$$
\bar{R}_{\alpha \beta}^{(h)}=-\frac{1}{2} \square_{\bar{g}} \bar{g}_{\alpha \beta}+S_{\alpha \beta}[\bar{g}, \partial \bar{g}]
$$

where $\square_{\bar{g}}$ is the scalar wave operator in $(\bar{M}, \bar{g})$. Hence the Einstein vacuum equations in spacetime harmonic coordinates is a quasi-linear hyperbolic

\footnotetext{
$2_{\text {for a tensor }} b$ on $M$, with $b_{i j}=b\left(e_{i}, e_{j}\right)$, we have $\mathcal{L}_{\partial_{t}} b_{i j}=\partial_{t}\left(b_{i j}\right)-b\left(\left[\partial_{t}, e_{i}\right], e_{j}\right)-$ $b\left(e_{i},\left[\partial_{t}, e_{j}\right]\right)$
} 
system and therefore the Cauchy problem ${ }^{3}$ for $\bar{R}_{\alpha \beta}^{(h)}=0$ is well posed and standard results give local existence. One proves that if the constraints and gauge conditions are satisfied initially, they are preserved by the evolution. This together with a Zorn's lemma argument gives the existence of a MVCD. Uniqueness is proved using the field equations to get a contradiction to the Haussdorff property, given a pair of non-isometric vacuum Cauchy developments, which are both maximal w.r.t. the natural partial ordering on the class of Cauchy developments.

A spacetime $(\bar{M}, \bar{g})$ is said to satisfy the time-like convergence condition (or strong energy condition) if

$$
\bar{R}_{a b} V^{a} V^{b} \geq 0, \quad \text { for all } V \text { with } \bar{g}_{a b} V^{a} V^{b} \leq 0 .
$$

Globally hyperbolic spacetimes with compact Cauchy surface and satisfying the time-like convergence condition are often called "cosmological spacetimes" in the literature, following [23. Here we will use the term spatially compact to refer to the existence of a compact Cauchy surface. A spacelike hypersurface $(M, g)$ in $(\bar{M}, \bar{g})$ has constant mean curvature if $\nabla_{i} \operatorname{tr}_{g} k=0$, cf. subsection 2.3 below.

We end this subsection with by stating a few facts about Killing fields.

Proposition 2.3 ([68). Let $M$ be a compact manifold and let $(M, g, k)$ be a constant mean curvature vacuum data set on $M$ with $\operatorname{MVCD}(\bar{M}, \bar{g})$. Let $Y$ be a Killing field on $(\bar{M}, \bar{g})$ and let $Y=Y_{\perp} T+Y_{\|}$be the splitting of $Y$ into its perpendicular and tangential parts at $M$. Then $\left(Y_{\perp}, Y_{\|}\right)$satisfy the conditions

(1) $Y_{\perp}=0, \mathcal{L}_{Y_{\|}} g=0, \mathcal{L}_{Y_{\|}} k=0$, in case $g$ is non-flat or $k \neq 0$.

(2) $Y_{\perp}$ is constant and $\mathcal{L}_{Y_{\|}} g=0$ if $g$ is flat and $k=0$.

On the other hand, given $Y_{\perp}, Y_{\|}$on $M$ satisfying conditions 1 , 2 above, there is a unique Killing field $Y$ on $\bar{M}$, with $Y=Y_{\perp} T+Y_{\|}$on $M$.

Proposition 2.4. Let $(\bar{M}, \bar{g})$ be a globally hyperbolic spacetime.

(1) Assume that $(\bar{M}, \bar{g})$ satisfies the time-like convergence condition and contains a compact Cauchy surface $M$ with constant mean curvature. Then either $(\bar{M}, \bar{g})$ is a metric product $M \times \mathbb{R}$ or any Killing field $Y$ on $(\bar{M}, \bar{g})$ is tangent to $M$. In particular, if $(\bar{M}, \bar{g})$ is vacuum and has a nonzero Killing not tangent to $M$, then $(\bar{M}, \bar{g})$ is flat.

(2) Assume a compact group $G$ acts by isometries on $(\bar{M}, \bar{g})$. Then the action of $G$ is generated by space-like Killing fields and $(\bar{M}, \bar{g})$ is foliated by Cauchy surfaces invariant under the action of $G$.

(3) Assume that $(\bar{M}, \bar{g})$ is $3+1$ dimensional. Let $M$ be a Cauchy surface in $\bar{M}$, let $Y$ be a Killing field on $\bar{M}$ and assume $Y$ is strictly spacelike, $\bar{g}(Y, Y)>0$, on $M$. Then $Y$ is strictly spacelike on $\bar{M}$.

\footnotetext{
${ }^{3}$ Note that the Einstein equations in spacetime harmonic gauge should be viewed as an evolution equation for $(g, k, N, X)$.
} 
Proof. Point 1 is a well known consequence of the uniqueness result for constant mean curvature hypersurfaces of Brill and Flaherty [35], cf. [124]. Point 2 is essentially [27, Lemma 1.1]. For the proof, note that as $G$ is compact we can construct a $G$ invariant time function on $\bar{M}$ by averaging any global time function $t$ on $\bar{M}$ w.r.t. the $G$ action, cf. the proof of [27, Lemma 1.1]. The level sets of the averaged time function are Cauchy surfaces and are invariant under the action of $G$. The result follows.

The following argument for point 3 is due to Alan Rendall ${ }^{4}$. Let $\mathcal{N}=$ $\{p \in \bar{M}: \bar{g}(Y, Y)=0\}$ and assume for a contradiction $\mathcal{N}$ is nonempty. Choose $p \in \mathcal{N}$ and a time function $t$ on $\bar{M}$ so that $t(M)=0$ and $t(p)>0$. Let $A$ denote the intersection of the past of $p$ with the future of $M$ and let $t_{1}=\inf \{t(q): q \in A \cap \mathcal{N}\}$. The set $\mathcal{N}$ is closed and by global hyperbolicity $A$ is compact and hence $t_{1}>0$ and there is a $q \in A \cap \mathcal{N}$ with $t(q)=t_{1}$. If $Y(q)$ is nonzero and null, then using the equation $Y^{a} \nabla_{a}\left(Y^{b} Y_{b}\right)=0$ which holds since $Y$ is Killing, gives a null curve of points in $\mathcal{N}$ where $Y$ is null. Following this into the past, shows that there is a $q \in \mathcal{N}$ with $t(q)<t_{1}$, which gives a contradiction. In case $Y(q)=0$, the linearization of $Y$ acts by isometries on $T_{q} \bar{M}$, and as the sphere of null directions in $T_{q} \bar{M}$ is two dimensional it leaves a null direction fixed. Using the exponential map shows that the action of $Y$ near $q$ leaves a null geodesic invariant, along which $Y$ must be zero or null. This leads to a contradiction as above.

2.1. Cosmic Censorship. Theorem 2.2 proves uniqueness of the MVCD of a given data set $(M, g, k)$. However, examples show that the MVCD may fail to be maximal in the class of all vacuum extensions, i.e. there exist examples of vacuum data sets $(M, g, k)$ with vacuum extensions $(\bar{M}, \bar{g})$ such that the MVCD of $(M, g, k)$ is a strict subset of $(\bar{M}, \bar{g})$.

Example 2.5. Consider the $n+1$-dimensional Minkowski space $\mathbb{R}^{n, 1}$ with metric $\eta=-d t^{2}+\left(d x^{1}\right)^{2}+\cdots+\left(d x^{n}\right)^{2}$, let $I^{+}(\{0\})$ be the interior of the future light cone. $I^{+}(\{0\})$ is globally hyperbolic with the hyperboloids as Cauchy surfaces, and with the mantle of the light cone as Cauchy horizon. Let $\Gamma$ be a cocompact discrete subgroup of the Lorentz group $\mathrm{SO}(n, 1)$. Then the quotient space $\bar{M}=\Gamma \backslash I^{+}(\{0\})$ is a globally hyperbolic, spatially compact spacetime. By choosing $\rho$ to be the Lorentzian distance from the origin, we get $\bar{g}=-d \rho^{2}+\rho^{2} \gamma$ where $\gamma$ is the standard hyperbolic metric on the compact quotient $M=\Gamma \backslash \mathbf{H}^{n} .(\bar{M}, \bar{g})$ is the $M V C D$ of the vacuum data set $(M, \gamma,-\gamma)$.

In case $n=1, \mathbf{H}^{1}=\mathbb{R}$, and a fundamental domain for $\Gamma$ can be found which intersects the null boundary of $I^{+}(\{0\})$ in an open interval. Therefore if $n=1$, there is a nontrivial extension of $\bar{M}$, which is still flat, but which fails to be globally hyperbolic, cf. figure 1. This spacetime is known as the Misner universe. The maximal extension is unique in this case.

\footnotetext{
${ }^{4}$ private communication, 1999
} 


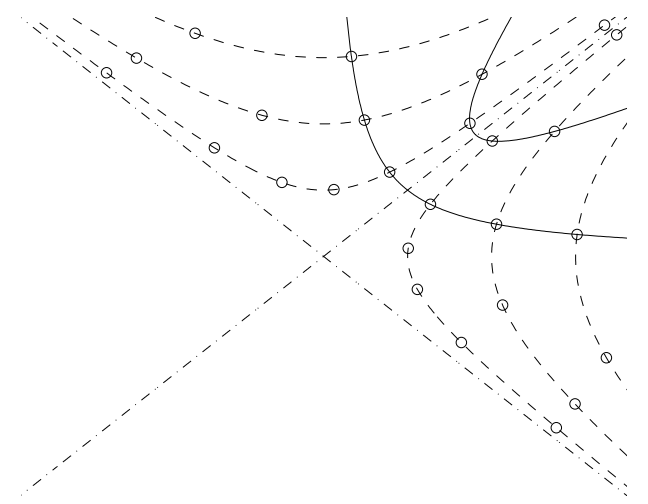

Figure 1. The $1+1$ dimensional Misner universe, showing a few orbits of $\mathrm{SO}(1,1)$ (dashed lines) and orbits of a discrete subgroup $\Gamma \subset \mathrm{SO}(1,1)$ (circles). The boundary of a fundamental domain for $\Gamma$ is plotted with solid lines.

If $n>1$, the ergodicity of the geodesic flow on $(M, \gamma)$ can be shown to prevent the existence of a spacetime extending $(\bar{M}, \bar{g})$ [104].

Higher dimensional examples of flat globally hyperbolic spatially compact spacetimes which admit nontrivial non-globally hyperbolic extensions can be constructed by taking products of the $n=1$ Misner universe with the flat torus.

A maximal vacuum extension may be non-unique, as is shown by the Taub-NUT example, cf. 53. If the MVCD of a vacuum data set is not a maximal vacuum extension, any extension of it must fail to satisfy the intuitively reasonable causality requirement of global hyperbolicity.

According to physical intuition, causality violations should be rare. This leads to the idea of cosmic censorship, essentially due to Penrose, see [133] for discussion. One way of stating this, relevant to the class of spacetimes we are concerned with here, is the following form of the strong cosmic censorship conjecture.

Conjecture 1 (Strong Cosmic Censorship). Let $M$ be a compact manifold of dimension 3. Then for generic vacuum data sets $(M, g, k)$, the maximal vacuum Cauchy development of $(M, g, k)$ is equal to the maximal vacuum extension of $(M, g, k)$.

In the case of asymptotically flat spacetimes (describing isolated systems in general relativity), the so-called weak cosmic censorship conjecture states that naked singularities (i.e. singularities which can be seen by an observer at infinity) should not occur generically, see the review paper by Wald [166] for a discussion of the status of the weak cosmic censorship conjecture. The work of Christodoulou, see [45] and references therein, see also the discussion in [166, §5], establishes weak cosmic censorship in the class of spherically symmetric Einstein-scalar field spacetimes, but also gives examples of initial 
data such that the Cauchy development has a naked singularity. For earlier surveys on the strong cosmic censorship conjecture, see [95] and 49.

The Penrose inequality, giving a lower bound on the ADM mass in terms of the area of a horizon in black hole spacetimes, was derived by a heuristic argument assuming the validity of the weak cosmic censorship conjecture. The proof of the Riemannian version of the Penrose inequality by Huisken and Ilmanen 91, 92, gives indirect support for the conjecture.

Let $(\bar{M}, \bar{g})$ be a spacetime and let $M \subset \bar{M}$ be a space-like hypersurface. The Cauchy horizon $H(M)$ is the boundary of the domain of dependence $D(M)$, cf. Appendix $\mathrm{A}$ for definition. If $M$ is compact without boundary, then every point of $\mathrm{H}^{+}(M)$ lies on a past inextendible null geodesic and every point of $H^{-}(M)$ lies on a future inextendible null geodesic, where $H^{+}(M)$ and $H^{-}(M)$ are the future and past components of $H(M)$, respectively.

Let $(\bar{M}, \bar{g})$ be a maximal vacuum extension of a vacuum data set $(M, g, k)$ with $M$ compact, and let $D(M) \subset \bar{M}$ be the MVCD of $(M, g, k)$. If $D(M) \neq$ $\bar{M}$, then the Cauchy horizon $H(M)$ is nonempty. One approach to SCC is to study the geometry of Cauchy horizons in vacuum spacetimes and to prove rigidity theorems as a consequence of extendibility of $D(M)$.

Isenberg and Moncrief proved for analytic vacuum or electrovac spacetimes, with analytic Cauchy horizon $H(M)$, that under the additional assumption that $H(M)$ is ruled by closed null geodesics, there is a nontrivial Killing field which extends to $D(M)$, see [100, 130. This result was generalized to the $C^{\infty}$ case by Friedrich et. al. [72]. As spacetimes with Killing fields are non-generic, this may be viewed as supporting evidence for the SCC.

In the class of Bianchi spacetimes (i.e. spatially locally homogenous spacetimes, cf. section (3), it has been proved by Chruściel and Rendall [55], generalizing work by Siklos [147] in the analytic case, that any $C^{\infty}$ Bianchi spacetime which contains a compact locally homogenous Cauchy horizon is a Taub spacetime, cf. section 3, (3.7) for definition. This result may be viewed as a version of SCC in the class of Bianchi spacetimes. In this context, it is worth mentioning that work by Chruściel and Galloway 52 gives examples which indicate that Cauchy horizons may be non-differentiable, generically.

Conjecture 2 (Bartnik [23, Conjecture 2]). Let $(\bar{M}, \bar{g})$ be a spatially compact globally hyperbolic spacetime satisfying the time-like convergence condition (2.5). Then if $(\bar{M}, \bar{g})$ is time-like geodesically complete, $(\bar{M}, \bar{g})$ splits isometrically as a product $\left(\mathbb{R} \times M,-d t^{2}+g\right)$.

If the Bartnik conjecture 2 is true, then any vacuum, globally hyperbolic, spatially compact spacetime, is either flat and covered by $\mathbb{R} \times T^{3}$ or is has an inextendible time-like geodesic which ends after a finite proper time, i.e. it is time-like geodesically incomplete. A sequence of points approaching the "end" of a finite length inextendible geodesic is often thought of as 
approaching a singularity. See [76] for a discussion of the status of the Bartnik conjecture.

Inextendibility of $D(M)$ can be detected by monitoring the asymptotic behavior of curvature invariants such as the Kretschmann scalar $\kappa$, defined by $\kappa=\bar{R}_{a b c d} \bar{R}^{a b c d}$. If $\kappa$ blows up along causal geodesics, then $D(M)$ fails to be extendible, and therefore proving blowup for $\kappa$ for generic spacetimes is an approach to proving SCC. This is the method used in the proof of cosmic censorship for the class of polarized Gowdy spacetimes [54, cf. section [4] and is likely to be important also in the cases with less symmetry. The structure of the horizon and extensions in the polarized Gowdy class can be very complicated as shown by the work of Chruściel et. al., see [49] for discussion, see also [53]. It was proved by Ringström, cf. Theorem 3.1, that for vacuum Bianchi spacetimes of class A, either the spacetime is Taub, cf. section [3, or $\kappa$ blows up at the singularity.

2.2. The evolution equations. A solution to the vacuum Einstein evolution equations with initial data is a curve $t \mapsto(g(t), k(t), N(t), X(t))$ defined on some interval $\left(T_{0}, T_{1}\right)$, satisfying (2.3).

Every regular solution $(g, k, N, X)$ to the vacuum evolution equations (2.3) with initial data solving the vacuum constraint equations, gives a vacuum spacetime. This is due to the fact that the constraint quantities

$$
\begin{aligned}
B & =R+(\operatorname{tr} k)^{2}-|k|^{2} \\
D_{i} & =\nabla_{i} \operatorname{tr} k-\nabla^{j} k_{i j}
\end{aligned}
$$

evolve according to a symmetric hyperbolic system and energy estimates together with an application of the Gronwall inequality allow one to show the the constraints are satisfied during the time of existence of the solution curve. Now the fact that the Einstein vacuum equation is equivalent to the system of constraint and evolution equations shows that the spacetime $(\bar{M}, \bar{g})$ constructed from the curve $(g, k, N, X)$ by letting $\bar{M}=\left(T_{0}, T_{1}\right) \times M$, and setting

$$
\bar{g}=-N^{2} d t^{2}+g_{i j}\left(d x^{i}+X^{i} d t\right)\left(d x^{j}+X^{j} d t\right)
$$

is a solution to the Einstein vacuum equations (2.1).

Note that in order for the solution to be well defined, it is necessary to specify the lapse and shift $(N, X)$, either as functions on spacetime $M \times$ $\left(T_{0}, T_{1}\right)$ or as functions of the data, $N=N[g, k], X=X[g, k]$; this may be viewed as a gauge fixing for the Einstein equations.

The choice of lapse and shift is crucial for the behavior of the solution curve. In particular, a foliation constructed for a particular choice of $N, X$ may develop singularities which are not caused by any singular or irregular nature of the Cauchy development. Consider for example the Gauss foliation condition $N=1, X=0$. Then the hypersurface $M$ flows in the direction of its unit normal and $M_{t}$ is simply the level set of the Lorentzian 
distance function $t(p)=d(M, p)$. The foliation $\left\{M_{t}\right\}$ will develop singularities precisely at the focal set of $M$, which in general will be nonempty, even in Minkowski space.

Many authors have considered hyperbolic reformulations of the Einstein equations, see the paper by Friedrich [71 for discussion, see also [73, 93, 94] for related work. The development of singularities for hyperbolic systems presents a serious obstacle to the numerical treatment of the Einstein evolution equations using hyperbolic reformulations, see 1, 2 for discussion and examples. It is therefore necessary to consider also gauges which make (2.3) into an elliptic-hyperbolic system.

2.3. Constant mean curvature foliations. A particularly interesting choice of gauge condition for the lapse function is given by the constant mean curvature (CMC) condition

$$
\nabla_{i} \operatorname{tr} k=0,
$$

i.e. the level sets $M_{t}$ of the time function $t$ are assumed to be hypersurfaces of constant mean curvature in $(\bar{M}, \bar{g})$. If $(\bar{M}, \bar{g})$ is globally hyperbolic, spatially compact and satisfies the time-like convergence condition (2.5), then for $\tau \in \mathbb{R}$, either there is at most one Cauchy surface with mean curvature trk $=\tau$ or $(\bar{M}, \bar{g})$ splits as a product, cf. 35.

This indicates that the mean curvature trk may be useful as a time function on $(\bar{M}, \bar{g})$ in the spatially compact case. Setting $t=\operatorname{tr} k$ leads, using (2.2 2.3) to the lapse equation

$$
-\Delta N+|k|^{2} N=1
$$

Local wellposedness for the Einstein evolution equations in $\mathrm{CMC}$ and more general elliptic time gauges given by a lapse equation of the form $-\Delta N+$ $|k|^{2} N=N T(h)$ for given spacetime functions $h$ has been proved by ChoquetBruhat and York 44. We may call this gauge the prescribed mean curvature (PMC) gauge. The proof is based on writing a wave equation for the second fundamental form, a technique which was also used in the work of ChoquetBruhat and Ruggeri [43] on harmonic time gauge. The prescribed mean curvature condition, has been used in the $\mathrm{U}(1) \times \mathrm{U}(1)$ symmetric case by Henkel [85] who proved global existence in this gauge. An important advantage of using PMC gauge is that one avoids the problem of proving existence of a CMC Cauchy surface.

Equation (2.6) supplemented by an elliptic shift gauge makes the Einstein evolution equations (2.3) into an elliptic-hyperbolic system of evolution equations. Let $\hat{g}$ be a given smooth metric on $M$, and let $V^{i}=g^{m n}\left(\Gamma_{m n}^{i}-\right.$ $\left.\hat{\Gamma}_{m n}^{i}\right)$. Then $-V^{i}$ is the tension field of the identity map Id $:(M, g) \rightarrow(M, \hat{g})$, and the $\mathrm{CMC}$ condition coupled with the spatially harmonic coordinate condition

$$
V^{i}=0
$$


gives a new elliptic gauge condition for the Einstein equations, CMCSH gauge [10. In 10, it is shown that the Einstein equations in CMCSH gauge forms an elliptic-hyperbolic system for the Einstein equations, which is well posed in $H^{s} \times H^{s-1}, s>n / 2+1$.

The maximal slicing condition trk $=0$ is of interest mainly for the asymptotically flat case. This was used in the proof of the nonlinear stability of Minkowski space by Christodoulou and Klainerman [46, cf. the discussion in section 6. Due to the "collapse of the lapse" phenomenon, see [25], the maximal foliation is not expected to cover the whole MVCD except in the small data case. See 124 for a discussion of maximal slices. Asymptotically flat spacetimes satisfying certain restrictions on the causal structure are known to contain maximal hypersurfaces [21]. The existence of CMC hypersurfaces in asymptotically flat spacetimes was considered in 8 .

The mean curvature operator satisfies a geometric maximum principle, see 7] for a proof of this under weak regularity. This allows one to use barriers to prove existence of constant mean curvature hypersurfaces. A spacetime is said to have crushing singularities if there are sequences of Cauchy surfaces with mean curvature trk tending uniformly to $\pm \infty$. Gerhardt 78 proved, using a barrier argument, that any spacetime satisfying (2.5) with crushing singularities is globally foliated by CMC hypersurfaces. These facts indicate that the CMC foliation condition is an interesting time gauge for the Einstein evolution equation. See also [79, 65, 22, 23, 85] for results relevant to existence of CMC hypersurfaces.

Let $R[g]$ be the scalar curvature. A $3-$ manifold $M$ is said to be of Yamabe type -1 if it admits no metric with $R[g]=0$ (and hence no metric with nonnegative scalar curvature), of Yamabe type 0 if it admits a metric with $R[g]=0$ but no metric with $R[g]=1$ and of Yamabe type +1 if it admits a metric with $R[g]=1$, cf. 67, Definition 9].

If the Cauchy surface $M$ is of Yamabe type -1, it follows from the constraint equation that $(\bar{M}, \bar{g})$ cannot contain a maximal (i.e. $\operatorname{tr} k=0)$ Cauchy surface and therefore one expects that (if the dominant energy condition holds) the maximal time interval of existence for (2.3) in CMC time is of the form (after a time orientation) $(-\infty, 0)$ with $\tau / 0$ corresponding to infinite expansion.

If $M$ is of Yamabe type 0 , then one expects that either the maximal CMC time interval is $(-\infty, 0)$ (possibly after a change of time orientation) or $(\bar{M}, \bar{g})$ splits as a product, and therefore in the vacuum case is covered by $\mathbb{R} \times T^{3}$ with the flat metric. Finally in case $M$ is of Yamabe type +1 , one expects the maximal CMC time interval to be $(-\infty, \infty)$, i.e. the spacetime evolves from a "big bang" to a "big crunch". This is formalized in the "closed universe recollapse conjecture" of Barrow, Galloway and Tipler [20].

Conjecture 3 (Constant mean curvature foliations). Let $M$ be a compact 3-manifold and let $(M, g, k)$ be a vacuum data set on $M$, with constant mean curvature. The Cauchy problem for the Einstein vacuum evolution equations 
with data $(M, g, k)$ has global existence in the constant mean curvature time gauge, i.e. there is a CMC foliation in the $\operatorname{MVCD}(\bar{M}, \bar{g})$ of $(M, g, k)$, containing $M$, with mean curvature taking all values in $(-\infty, \infty)$ in case $M$ has Yamabe type +1 and in case $M$ has Yamabe type 0 or -1, taking all values in $(-\infty, 0)$ (possibly after a change of time orientation).

Remark 2.1. Conjecture 3 has been stated in essentially this form by Rendall 135, Conjecture 1], see also Eardley and Moncrief [129, Conjecture C2] for a closely related statement.

Note that as $\tau \searrow-\infty$, the past focal distance of the (unique) CMC surface with mean curvature $\tau$ tends to zero, and hence the foliation exhausts the past of $M$. It follows that in case $M$ has Yamabe type +1 , then if Conjecture 3 is true, $(\bar{M}, \bar{g})$ is globally foliated by $C M C$ hypersurfaces. In case $M$ has Yamabe type 0 or -1 on the other hand, there is the possibility that the CMC foliation does not cover all of $\bar{M}$, due to the fact that as the mean curvature $\tau \nearrow 0$, the CMC hypersurfaces are expected to avoid black holes, by analogy with the behavior of CMC and maximal hypersurfaces in the Schwarzschild spacetime. See [135] for further remarks and conjectures related to this.

If one were able to prove Conjecture 3, then as remarked in [129], this would give the possibility of attacking the Cosmic Censorship Conjecture using PDE methods. There are no known counterexamples to Conjecture 3 for vacuum spacetimes. However, Isenberg and Rendall [103] give an example of dust spacetimes, not covered by a CMC foliation. Bartnik 23] gave an example of a spatially compact, globally hyperbolic spacetime satisfying the time-like convergence condition, which contains no CMC Cauchy surface. It is an interesting open question whether or not similar counter examples are possible in the vacuum case. The gluing results for solutions of the constraint equations, see [97, 98, 58, may lead to the construction of such examples.

The CMC conjecture 3 has been proved in a number of cases for spacetimes with symmetry, in the sense of the existence of a group $G$ of isometries acting (locally) on $(\bar{M}, \bar{g})$ by isometries and with space-like orbits. In the case of Bianchi IX, cf. section [3] which has Yamabe type +1 , the closed universe recollapse conjecture and consequently Conjecture 3, was proved by $\operatorname{Lin}$ and Wald 121. In the case of 2+1-dimensional vacuum spacetimes with cosmological constant, the conclusion of Conjecture 3 is valid [11. In the $2+1$ case, the CMC foliations are global.

The work of Rendall and Burnett, see [36] and references therein, proves under certain restrictions on the matter that a maximal, globally hyperbolic, spherically symmetric spacetime, which contains a CMC Cauchy surface diffeomorphic to $S^{2} \times S^{1}$, is globally foliated by CMC hypersurfaces with mean curvature taking on all real values.

We end this subsection by mentioning the harmonic time gauge condition, $\square_{\bar{g}} t=0$ or

$$
\bar{g}^{a b} \bar{\Gamma}_{a b}^{0}=0
$$


In case $X=0$, this is equivalent to the condition $N=\sqrt{\operatorname{det} g} / \sqrt{\operatorname{det} e}$ where $e_{i j}$ is some fixed Riemannian metric on $M$. The Einstein evolution equations with $X=0$ were proved to be hyperbolic with this time gauge by Choquet-Bruhat and Ruggeri [43. This time gauge also appears in the work of Gowdy and is used in the analysis of the Gowdy spacetimes as well as in the numerical work of Berger, Moncrief et. al. on Gowdy and U(1) spacetimes, cf. sections 4 and 5 .

The papers of Smarr and York [148, 149] contain an interesting discussion of gauge conditions for the Einstein equations. See also section [6 for a discussion of the spatial harmonic coordinate gauge and the survey of Klainerman and Nicolo [110, for further comments on gauges.

2.4. The Einstein equations as a system of quasi-linear PDE's. As mentioned above, the Einstein vacuum equations in spacetime harmonic coordinates form a quasi-linear hyperbolic system of the form

$$
-\frac{1}{2} \square_{\bar{g}} \bar{g}_{\alpha \beta}+S_{\alpha \beta}[\bar{g}, \partial \bar{g}]=0 .
$$

The system (2.7) is a quasi-linear wave equation, quadratic in the first order derivatives $\partial \bar{g}$ and with top order symbol depending only on the field $\bar{g}$ itself. Standard results show that the Cauchy problem is well posed in Sobolev spaces $H^{s} \times H^{s-1}, s>n / 2+1$. This was first proved for the Einstein equations by Hughes, Kato and Marsden [90]. It is also possible to prove this for elliptic-hyperbolic systems formed by the Einstein evolution equations together with the CMC-spatial harmonic coordinates gauge, see [10], see also section 6 for a discussion of the spatial harmonic coordinates gauge.

Recent work using harmonic analysis methods by Bahouri and Chemin 18, Klainerman and Rodnianski [115, 114, 113] and Smith and Tataru [151, see also [157] has pushed the regularity needed for systems of the above type on $\mathbb{R}^{n, 1}$ to $s>(n+1) / 2$ for $n \geq 3$. In order to get well posedness for $s$ lower than the values given above, it is likely one needs to exploit some form of the null condition, [154, 152, 157]. The null condition for equations of the form $\square_{\eta} u=F[u, \partial u]$ on Minkowski space states roughly that the symbol of the non-linearity $F$ cancels null vectors. For a discussion of the null condition on a curved space background, see [153, 150. Counter examples to well posedness for quasi-linear wave equations with low regularity data are given by Lindblad, see 122 .

The standard example of an equation which satisfies the null condition is $\square u=\partial_{\alpha} u \partial_{\beta} u \eta^{\alpha \beta}$ where $\square$ is the wave operator w.r.t. the Minkowski metric $\eta$ on $\mathbb{R}^{n, 1}$. This equation is well posed for data in $H^{s} \times H^{s-1}$ with $s>n / 2$ [116, and has global existence for small data for $n \geq 3$. On the other hand, the equation $\square u=\left(\partial_{t} u\right)^{2}$ which does not satisfy the null condition can be shown to have a finite time of existence for small data in the $3+1$ dimensional case. 
For quasi-linear wave equations which satisfy an appropriate form of the null condition [89, global existence for small data is known in $3+1$ dimensions. The Einstein equations, however, are not known to satisfy the null condition in any gauge. In particular, it can be seen that in spacetime harmonic coordinates, the Einstein equations do not satisfy the null condition. However, the analysis by Blanchet and Damour 34 of the expansion of solutions of Einstein equations in perturbation series around Minkowski space indicates that the logarithmic terms in the gravitational field in spacetime harmonic coordinates, arising from the violation of the null condition, can be removed after a (nonlocal) gauge transformation to radiative coordinates where the coordinate change depends on the history of the field. A similar analysis can be done for the Yang-Mills (YM) equation in Lorentz gauge. It may further be argued that the small data, global existence proof of Christodoulou and Klainerman for the Einstein equations exploits properties of the Einstein equations related to the null condition. In a recent paper, Lindblad and Rodnianski [123. have introduced a weak form of the null condition and shown that the Einstein equations satisfy this.

Global existence is known for several of the classical field equations such as certain nonlinear Klein-Gordon (NLKG) equations and the YM equation on $\mathbb{R}^{3,1}$ (proved by Eardley and Moncrief, 63, 64). The proofs for NLKG and the proof of Eardley and Moncrief for YM use light cone estimates to get apriori $L^{\infty}$ bounds. The proof of Eardley and Moncrief used the special properties of YM in the radial gauge. This method was also used in the global existence proof for YM on 3+1-dimensional, globally hyperbolic spacetimes by Chruściel and Shatah [56. Klainerman and Machedon [109] were able to prove that the YM equations on $\mathbb{R}^{3,1}$ in Coloumb gauge satisfy a form of the null condition and are well posed in energy space $H^{1} \times L^{2}$. They were then able to use the fact that the energy is conserved to prove global existence for YM. See also [108 for an overview of these ideas and some related conjectures.

An important open problem for the classical field equations is the global existence problem for the wave map equation (nonlinear $\sigma$-model, hyperbolic harmonic map equation). This is an equation for a map $\mathbb{R}^{n, 1} \rightarrow N$, where $N$ is some complete Riemannian manifold,

$$
\square_{\eta} u^{A}+\Gamma_{B C}^{A}(u) \partial_{\alpha} u^{B} \partial_{\beta} u^{C} \eta^{\alpha \beta}=0 .
$$

Here $\Gamma$ is the Christoffel symbol on $N$.

The wave map equation satisfies the null condition and hence we have small data global existence for $n \geq 3$. Small data global existence is also known for $n=2$. Further, scaling arguments provide counterexamples to global existence for $n \geq 3$, whereas $n=2$ is critical with respect to scaling. For $n \geq 2$, global existence for "large data" is known only for symmetric solutions, and in particular, the global existence problem for the wave map equation (2.8) is open for the case $n=2$. For the case $n=1$, global existence can be proved using energy estimates or light cone estimates. Tao proved 
[156] that the wave map equation in $2+1$ dimensions with the sphere as target is globally well-posed for small energy. The proof is based on a new local well-posedness in $H^{1} \times L^{2}$ and conservation of energy. This result has been generalized to the case of hyperbolic target by Krieger 119. Tataru 158 has proved that the wave map equation in $n+1$ dimensions is locally well-posed in $\dot{H}^{n / 2} \times \dot{H}^{n / 2-1}$ for general target imbedded in $\mathbb{R}^{m}$. (Here $\dot{H}^{s}$ is the homogenous Sobolev space. See [158] for the precise definition of well-posedness used). See also [146, 159] for surveys.

The above discussion shows that the situation for the wave map equation is reminiscent of that for the Einstein equations, cf. sections 4, 5. In particular, it is interesting to note that equations of the wave map type show up in the reduced vacuum Einstein equations for the Gowdy and U(1) problems.

\section{BIANCHI}

Let $(\bar{M}, \bar{g})$ be a $3+1$ dimensional spacetime with 3-dimensional local isometry group $G$. Assume the action of $G$ is generated by space-like Killing fields and that the orbits of $G$ in the universal cover of $\bar{M}$ are 3-dimensional. This means there is a global foliation of $\bar{M}$ by space-like Cauchy surfaces $M$ with locally homogeneous induced geometry. Such spacetimes are known as Bianchi spacetimes. The assumption of local homogeneity of the $3-$ dimensional Cauchy surfaces means that a classification of the universal cover is given by the classification of 3 -dimensional Lie algebras.

Let $e_{a}, a=0, \ldots, 3$ be an ON frame on $\bar{M}$, with $e_{0}=u$, a unit time-like normal to the locally homogeneous Cauchy surfaces, let $\gamma_{a b}^{c}$ be the commutators of the frame, $\left[e_{a}, e_{b}\right]=\gamma_{a b}^{c} e_{c}$. Let the indices $i, j, k, l$ run over $1,2,3$. We may without loss of generality assume that $\left[e_{a}, \xi_{i}\right]=0$ where $\left\{\xi_{i}\right\}_{i=1}^{3}$ is a basis for the Lie algebra $\mathfrak{g}$ of $G$.

Choose a time function $t$ so that $t_{, a} u^{a}=1$, i.e. the level sets of $t$ coincide with the group orbits. Restricting to a level set $M$ of $t$, the spatial part of the commutators $\gamma_{i j}^{k}$ are the structure constants of $\mathfrak{g}$. These can be decomposed into a constant symmetric matrix $n^{k l}$ and a vector $a_{i}$,

$$
\gamma_{i j}^{k}=\epsilon_{i j l} n^{k l}+a_{i} \delta_{j}^{k}-a_{j} \delta_{i}^{k} .
$$

We will briefly describe the classification used in the physics literature, cf. [66], [163, §1.5.1].

The Jacobi identity implies $n^{i j} a_{j}=0$ and by choosing the frame $\left\{e_{i}\right\}$ to diagonalize $n^{i j}$ and so that $e_{1}$ is proportional to the vector $a_{i}$, we get

$$
n^{i j}=\operatorname{diag}\left(n_{1}, n_{2}, n_{3}\right), \quad a_{i}=(\mathbf{a}, 0,0) .
$$

The 3-dimensional Lie algebras are divided into two classes by the condition $\mathbf{a}=0(\operatorname{class} \mathrm{A})$ and $\mathbf{a} \neq 0$ (class $\mathrm{B})$. The classes $A$ and $B$ correspond in mathematical terminology to the unimodular and non-unimodular Lie algebras. If $n_{2} n_{3} \neq 0$, let the scalar $h$ be defined by

$$
\mathbf{a}^{2}=h n_{2} n_{3} .
$$


TABLE 1. Bianchi geometries

\begin{tabular}{|c|c|c|c|c|c|c|c|c|c|}
\hline & Type & $n_{1}$ & $n_{2}$ & $n_{3}$ & \multirow{7}{*}{ Class B } & & & & \\
\hline & I & 0 & 0 & 0 & & Type & $n_{1}$ & $n_{2}$ & $n_{3}$ \\
\hline & II & 0 & 0 & + & & $\mathrm{V}$ & 0 & 0 & 0 \\
\hline \multirow[t]{4}{*}{ Class A } & $\mathrm{VI}_{0}$ & 0 & - & + & & IV & 0 & 0 & + \\
\hline & $\mathrm{VII}_{0}$ & 0 & + & + & & $\mathrm{VI}_{h}$ & 0 & - & + \\
\hline & VIII & - & + & + & & $\mathrm{VII}_{h}$ & 0 & + & + \\
\hline & IX & + & + & + & & & & & \\
\hline
\end{tabular}

Table 1 gives the classification of Bianchi geometries. Note that the invariance of the Bianchi types under permutations and sign changes of the frame elements has been used to simplify the presentation. Here the notation $\mathrm{VI}_{0}$, $\mathrm{VII}_{0}, \mathrm{VI}_{h}, \mathrm{VII}_{h}$ refers to the value of $h$ defined by (3.2). In the list of Bianchi types I-IX, the missing type III is the same as $\mathrm{VI}_{-1}$.

Due to the local homogeneity of the Cauchy surfaces $M$ in a Bianchi spacetime, the topologies of the spatially compact Bianchi spacetimes can be classified using the classification of compact manifolds admitting Thurston geometries.

The eight Thurston geometries $S^{3}, E^{3}, H^{3}, S^{2} \times \mathbb{R}, H^{2} \times \mathbb{R}$, Nil, $\widetilde{\mathrm{SL}}(2, \mathbb{R})$, Sol, are the maximal geometric structures on compact 3-manifolds, see 145, 160] for background. Each compact 3-manifold with a Bianchi (minimal) geometry also admits a Thurston (maximal) geometry, and this leads to a classification of the topological types of compact 3-manifolds with Bianchi geometry, i.e. compact manifolds of the form $X / \Gamma$ where $X$ is a complete, simply connected 3 -manifold with a Bianchi geometry and $\Gamma$ is a cocompact subgroup of the isometry group of $X$. It is important to note that $\Gamma$ is not always a subgroup of the 3-dimensional Bianchi group $G$.

The relation between the Bianchi types admitting a compact quotient and the Thurston geometries is given by Table 2. For each Bianchi type we give only the maximal Thurston geometries corresponding to it, see [74, 117, 118. for further details and references. We make the following remarks

Remark 3.1. (i) Let $(M, g)$ be a 3-dimensional space form with sectional curvature $\kappa=-1,0,+1$. A spacetime $(\bar{M}, \bar{g})$ with $\bar{M}=$ $M \times(a, b)$ and a warped product metric $\bar{g}=-d t^{2}+w^{2}(t) g$, satisfying the perfect fluid Einstein equations is called a (local) FriedmannRobertson-Walker (FRW) spacetime. Specifying the equation of state for the matter in the Einstein equations leads to an ODE for $w$. The FRW spacetimes play a central role in the standard model of cosmology. In the vacuum case, only $\kappa=-1,0$ are possible, and in this case, the spatially compact local FRW spacetimes are for $\kappa=0$, the flat spacetimes covered by $T^{3} \times \mathbf{E}^{1}$, a special case of Bianchi I, and for $\kappa=-1$, the local FRW spacetimes discussed in example 2.5. which are Bianchi $V$. 
TABle 2. Bianchi and Thurston geometries

\begin{tabular}{|c|c|c|c|}
\hline Bianchi type & class & Thurston geometry & comments \\
\hline $\mathrm{I}$ & $\mathrm{A}$ & $\mathbf{E}^{3}$ & \\
\hline II & $\bar{A}$ & Nil & \\
\hline $\mathrm{III}=\mathrm{VI}_{-1}$ & B & $\begin{array}{l}\mathbf{H}^{2} \times \mathbf{E}^{1} \\
\widetilde{\mathrm{SL}}(2, \mathbb{R})\end{array}$ & cf. Remark 3.1] iii] \\
\hline IV & E & - & no compact quotient \\
\hline $\mathrm{V}$ & $\mathrm{B}$ & $\mathbf{H}^{3}$ & cf. Remark 3.1]iv \\
\hline $\mathrm{VI}_{0}$ & $\bar{A}$ & Sol & \\
\hline $\mathrm{VI}_{h}, h \neq 0,-1$ & - & - & no compact quotient \\
\hline $\mathrm{VII}_{0}$ & $\mathrm{~A}$ & $\mathbf{E}^{3}$ & \\
\hline $\mathrm{VII}_{h}, h \neq 0$ & $\mathrm{~B}$ & $\mathbf{H}^{3}$ & cf. Remark [3.1]iv \\
\hline VIII & $\bar{A}$ & $\widehat{\mathrm{SL}}(2, \mathbb{R})$ & \\
\hline IX & $\bar{A}$ & $S^{3}$ & \\
\hline
\end{tabular}

(ii) The Thurston geometry $S^{2} \times \mathbb{R}$ admits no 3-dimensional group of isometries, and hence it does not correspond to a Bianchi geometry, but to a Kantowski-Sachs geometry [105] with symmetry group $\mathrm{SO}(3) \times \mathbb{R}$. The other cases with 4-dimensional symmetry group are vacuum LRS Bianchi I, II, III, VIII and IX, see [66, p. 133].

(iii) The type of geometry depends on the subgroup $\Gamma$ of the isometry group used to construct the compactification. The isometry group in turn depends on the Bianchi data. Note that $\widetilde{S L}(2, \mathbb{R})$ is both maximal and minimal, whereas the isometry group of $\mathbf{H}^{2} \times \mathbb{R}$ has dimension 4 and is therefore not a minimal (Bianchi) group.

(iv) The compactifications of Bianchi $V$ and $V I I_{h}, h \neq 0$ are both of the type discussed in Example [2.5, thus no anisotropy is allowed in the compactification of Bianchi type $V$ and $V I I_{h}, h \neq 0$.

(v) The compactification of a Bianchi geometry, introduces new (moduli) degrees of freedom, in addition to the dynamical degrees of freedom, see [117] and references therein for discussion, see also [50]. The resulting picture is complicated and does not appear to have been given a definite treatment in the literature.

In the rest of this section, we will concentrate on class A Bianchi spacetimes. We will also refrain from considering the moduli degrees of freedom introduced by the compactification, as it can be argued that these are not dynamical.

Let the expansion tensor $\theta_{i j}$ be given by

$$
\theta_{i j}=\nabla_{j} u_{i}
$$

(i.e. $\theta_{i j}=-k_{i j}$ where $k_{i j}$ is the second fundamental form). Decompose $\theta_{i j}$ as $\theta_{i j}=\sigma_{i j}+H \delta_{i j}$ where $H=\theta / 3, \theta=g^{i j} \theta_{i j} / 3$. Then $H$ is the Hubble 
scalar. We are assuming (3.1) and in the vacuum class A case it follows that $\sigma_{i j}$ is diagonal [163, p. 41].

Under these assumptions, $\gamma_{a b}^{c}=\gamma_{a b}^{c}(t)$ and we may describe the geometry of $(\bar{M}, \bar{g})$ completely in terms of $\gamma_{a b}^{c}$ or equivalently in terms of the 3 -dimensional commutators $\gamma_{i j}^{k}$ and the expansion tensor given in terms of $\sigma_{i j}, \theta$.

By a suitable choice of frame, $\sigma_{i j}$ and $n_{i j}$ can be assumed diagonal. Since $\sigma_{i j}$ is traceless and diagonal, it can be described in terms of the variables

$$
\sigma_{+}=\frac{1}{2}\left(\sigma_{22}+\sigma_{33}\right), \quad \sigma_{-}=\frac{1}{2 \sqrt{3}}\left(\sigma_{22}-\sigma_{33}\right)
$$

and similarly, $n_{i j}$ can be represented by

$$
\left(n_{1}, n_{2}, n_{3}\right)=\frac{1}{2 \sqrt{3}}\left(n_{11}, n_{22}, n_{33}\right)
$$

The curvature $b_{i j}=R i c\left(e_{i}, e_{j}\right)$ can be written in terms of $n_{i j}, b_{i j}=2 n_{i}{ }^{k} n_{k j}-$ $n_{k}{ }^{k} n_{i j}$ and is therefore diagonal in the chosen frame. Decomposing $b_{i j}$ into trace-free and trace parts $s_{i j}, k$ we find that $s_{i j}$ can be represented by two variables $s_{+}, s_{-}$.

Next we introduce the dimensionless variables (following Hsu and Wainwright [164, but normalizing with $H$ instead of $\theta$ ),

$$
\left(\Sigma_{+}, \Sigma_{-}, N_{1}, N_{2}, N_{3}\right)=\left(\sigma_{+}, \sigma_{-}, n_{1}, n_{2}, n_{3}\right) / H
$$

Similarly, we set

$$
\left(S_{+}, S_{-}\right)=\left(s_{+}, s_{-}\right) / H^{2}
$$

In addition to these choices we also define a new time $\tau$ by $e^{\tau}=\ell, \ell$ the length scale factor, or $d t / d \tau=1 / H$.

Clearly the equations are invariant under permutations $\left(\Sigma_{i}\right) \rightarrow P\left(\Sigma_{i}\right)$, $\left(N_{i}\right) \rightarrow P\left(N_{i}\right)$. Cyclic permutations of $\left(N_{i}\right),\left(\Sigma_{i}\right)$ correspond to rotations through $2 \pi / 3$ in the $\Sigma_{+}, \Sigma_{-}$plane.

We now specialize to the vacuum case. Then (for $H \neq 0$ ) the Einstein equations are equivalent to the following system of ODE's for $\Sigma_{+}, \Sigma_{-}, N_{1}$, $N_{2}, N_{3}$ (where the' denotes derivative w.r.t. the time coordinate $\tau$ ):

$$
\begin{aligned}
N_{1}^{\prime} & =\left(q-4 \Sigma_{+}\right) N_{1}, \\
N_{2}^{\prime} & =\left(q+2 \Sigma_{+}+2 \sqrt{3} \Sigma_{-}\right) N_{2}, \\
N_{3}^{\prime} & =\left(q+2 \Sigma_{+}-2 \sqrt{3} \Sigma_{-}\right) N_{3}, \\
\Sigma_{+}^{\prime} & =-(2-q) \Sigma_{+}-3 S_{+}, \\
\Sigma_{-}^{\prime} & =-(2-q) \Sigma_{-}-3 S_{-},
\end{aligned}
$$


where

$$
\begin{aligned}
q & =2\left(\Sigma_{+}^{2}+\Sigma_{-}^{2}\right), \\
S_{+} & =\frac{2}{3}\left[\left(N_{2}-N_{3}\right)^{2}-N_{1}\left(2 N_{1}-N_{2}-N_{3}\right)\right], \\
S_{-} & =\frac{2}{\sqrt{3}}\left(N_{3}-N_{2}\right)\left(N_{1}-N_{2}-N_{3}\right) .
\end{aligned}
$$

The Hamiltonian constraint (2.2a) is in terms of these variables

$$
\Sigma_{+}^{2}+\Sigma_{-}^{2}+\left[N_{1}^{2}+N_{2}^{2}+N_{3}^{2}-2\left(N_{1} N_{2}+N_{2} N_{3}+N_{3} N_{1}\right)\right]=1 .
$$

With our conventions, if $\left(\tau_{-}, \tau_{+}\right)$is the maximal time interval of existence for the solution to (3.3), then $\tau \rightarrow \tau_{-}$corresponds to the direction of a singularity. For all non-flat vacuum Bianchi models except Bianchi IX, the spacetime undergoes an infinite expansion as $\tau \rightarrow \tau_{+}$and is geodesically complete in the expanding direction, cf. [134, Theorem 2.1] which covers the vacuum case as a special case. For Bianchi IX, on the other hand, $\tau \rightarrow \tau_{+}$corresponds to $H \rightarrow 0$, as follows from the proof of the closed universe recollapse conjecture for Bianchi IX by Lin and Wald 121, and hence to the dimensionless variables becoming ill defined.

We will now review the basic facts for Bianchi types I, II and IX.

I: Kasner. The Hamiltonian (3.4) constraint reads

$$
\Sigma_{+}^{2}+\Sigma_{-}^{2}=1
$$

and the induced metric on each time slice is flat. These spacetimes can be given metrics of the form

$$
d s^{2}=-d t^{2}+\sum_{i} t^{2 p_{i}} d x^{i} \otimes d x^{i}
$$

where $\sum_{i} p_{i}=1, \sum_{i} p_{i}^{2}=1$, the Kasner relations, which correspond to the equation (3.5). The $\Sigma_{ \pm}$and $p_{i}$ are related by $\Sigma_{+}=\frac{3}{2}\left(p_{2}+p_{3}\right)-1$, $\Sigma_{-}=\frac{\sqrt{3}}{2}\left(p_{2}-p_{3}\right)$. Clearly, the Kasner circle given by (3.5) consists of fixed points to the system (3.3). The points $T_{1}, T_{2}, T_{3}$, with coordinates $(-1,0)$, $(1 / 2, \pm \sqrt{3} / 2)$ correspond to flat spacetimes of type I or $\mathrm{VII}_{0}$ (quotients of Minkowski space).

II: By using the permutation symmetry of the equations, we may assume $N_{2}=N_{3}=0$. The solution curve which is a subset of the cylinder $\Sigma_{+}^{2}+\Sigma_{-}^{2}<$ 1 has a past endpoint on the longer arc connecting $T_{2}$ and $T_{3}$ and future endpoint on the shorter arc connecting these points see figure 2. This curve realizes the so called Kasner map, cf. [163, §6.4.1]. We define the Bianchi II variety as the union of the three spheres in $\mathbb{R}^{5}$ given by the solutions to the constraint (3.4) with $\left(N_{2}, N_{3}\right)=0$ and permutations thereof. The Kasner circle is the intersection of the spheres. 


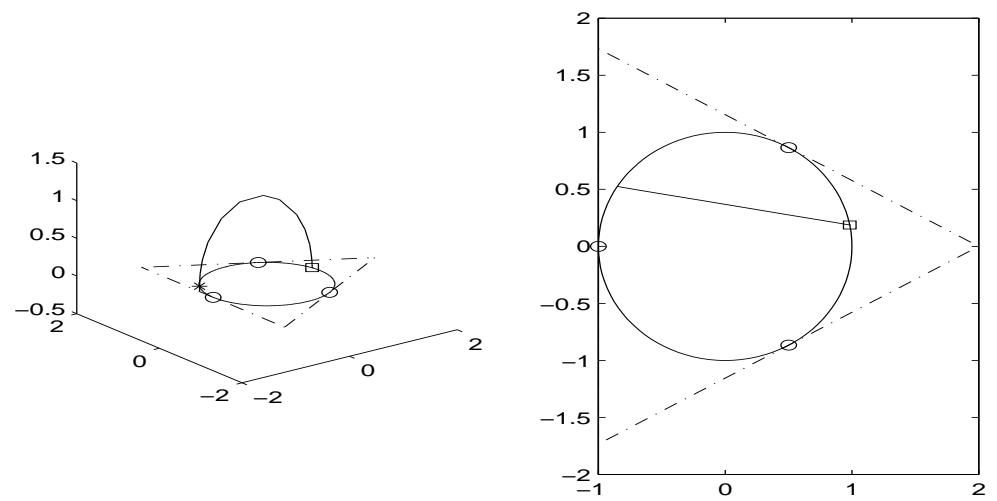

Figure 2. A type II solution, the Kasner circle and the triangle for the Kasner billiard in the $\left(\Sigma_{+}, \Sigma_{-}\right)$-plane are shown.

IX: Mixmaster, characterized by $N_{i}>0, i=1,2,3$. The heuristic picture is the following. The projection in the $\left(\Sigma_{+}, \Sigma_{-}\right)$-plane of a generic orbit in the direction $\tau \searrow \tau_{-}$moves into the Kasner circle and stays there, undergoing an infinite sequence of bounces, which are approximately given by the Kasner billiard, cf. figure 4. This picture is supported by numerical studies of the full Bianchi IX system, see eg. 30.

The Kasner billiard is the dynamical system given by mapping a (nonflat) point $p$ on the Kasner circle to the point on the Kasner circle which is the end point of the type II orbit starting at $p$. This map can be described as follows. Let $B$ be the nearest corner to $p$ of the triangle shown in figure 4. The ray starting at $B$ through $p$ intersects the Kasner circle in a point $q$, which is the image of $p$ under the Kasner map, see also [163, Fig. 6.13]. Iterating this construction gives a sequence of points $\left\{p_{i}\right\}$ on the Kasner circle, which we may call the Kasner billiard.

The exceptional orbits which do not exhibit this infinite sequence of bounces are the Taub type IX solutions, cf. figure 3. Up to a permutation these are given by the conditions

$$
N_{2}=N_{3}, \quad \Sigma_{-}=0
$$

We call a Bianchi spacetime, satisfying (3.7) up to a permutation, a Taub spacetime.

The past limit of the Taub type IX solution is the flat point $(-1,0)$. The MVCD of Taub type IX data has a smooth Cauchy horizon, and is extendible, the extension being given by the so-called Taub-NUT spacetimes. As shown by Chruściel and Rendall [55. Theorem 1.2], these are the only Bianchi IX spacetimes with a smooth Cauchy horizon, which gives a version of SCC for this class. See [55] for further details on the status of SCC in the locally homogeneous case. Chruściel and Isenberg proved that the MVCD of 

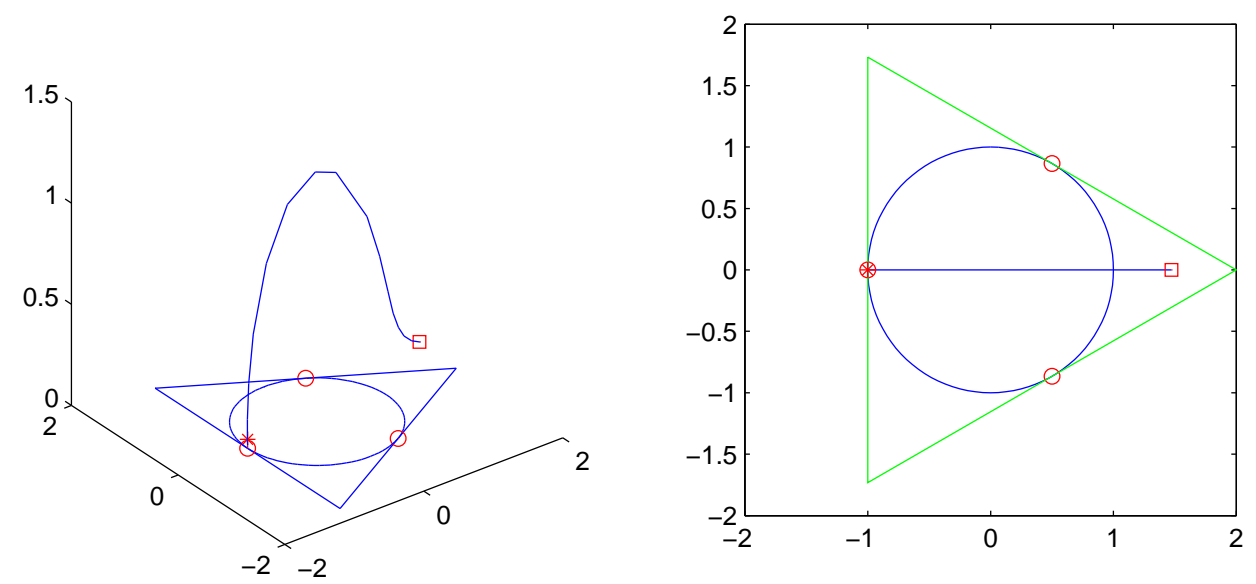

Figure 3. A Taub type IX solution, the Kasner circle and the triangle for the Kasner billiard in the $\left(\Sigma_{+}, \Sigma_{-}\right)$-plane are shown.

Taub type IX data has non-isometric maximal vacuum extensions, further emphasizing its pathological nature, cf. [53].

From the point of view of the cosmic censorship conjecture, the following theorem appears fundamental. A point which is a past limit point of $\left(\Sigma_{+}(\tau), \Sigma_{-}(\tau), N_{1}(\tau), N_{2}(\tau), N_{3}(\tau)\right)$ is called an $\alpha$ limit point. We say that the approach to the singularity is oscillatory, if the set of $\alpha$ limit points contains at least two points on the Kasner circle, at least one of which is disctinct from the special points $T_{1}, T_{2}, T_{3}$, cf. [137.

Theorem 3.1 (137, 141]). A vacuum Bianchi spacetime of class A has exactly one of the properties

(1) The Kretschmann scalar $\kappa=\bar{R}_{\alpha \beta \gamma \delta} \bar{R}^{\alpha \beta \gamma \delta}$ satisfies $\lim \sup _{\tau} \searrow \tau_{-}|\kappa|=$ $\infty$

(2) The MVCD has a smooth Cauchy horizon and the spacetime is a Taub spacetime.

For non-Taub vacuum Bianchi VIII and IX spacetimes, the approach to the singularity is oscillatory.

Remark 3.2. $\quad$ (i) Rendall [137] proved the dichotomy in Theorem [3.1] for all Bianchi class A except VIII and IX. These cases as well as the oscillatory behavior for type VIII and IX were proved by Ringstrom [141. Curvature blowup for non-Taub Bianchi A models with perfect fluid matter, including stiff fluid was proved by by Ringstrom 142.

(ii) In class $B$ it is only the exceptional model Bianchi $V I_{-1 / 9}$ which exhibits oscillatory behavior as has been argued by Hewitt et. al [87.

(iii) Theorem [3.1 shows that SCC holds in the class of vacuum Bianchi class A spacetimes, also with respect to $C^{2}$ extensions. 


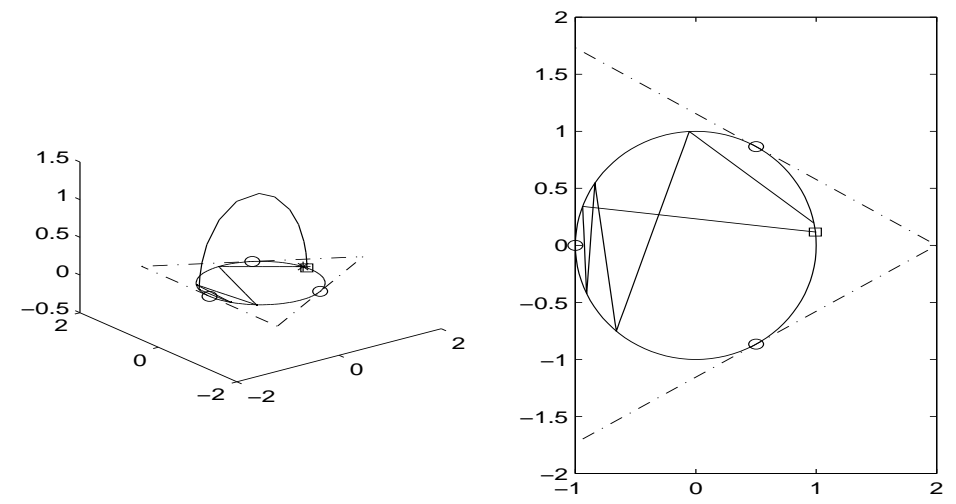

FIGURE 4. Bianchi IX orbit showing a few bounces. The vertical axis is $N_{1}$. The Kasner circle and the triangle for the Kasner billiard in the $\left(\Sigma_{+}, \Sigma_{-}\right)$-plane are shown.

(iv) See also Weaver [167] for a related result for Bianchi $V I_{0}$ with a magnetic field. Weaver proved that the singularity in magnetic Bianchi $V I_{0}$ is oscillatory and that curvature blows up as one approaches the singularity. It should be noted that vacuum Bianchi $V I_{0}$, on the other hand, is non-oscillatory.

The dynamics of the Bianchi spacetimes has been studied for a long time, from the point of view of dynamical systems. In particular, it is believed that the Bianchi IX (mixmaster) solution is chaotic in some appropriate sense, see for example the paper by Hobill in [163] or the collection 88 as well as the work of Cornish and Levin [57, for various points of view. However, it does not yet appear to be clear which is the appropriate definition of chaos to be used, and no rigorous analysis exists for the full Bianchi IX system. In the course of the above mentioned work, approximations to the Bianchi dynamics have been described and studied, such as the Kasner billiard (cf. fig. 4, and the discussion above) and the BKL map, cf. [163, §11.2.3].

It has long been conjectured that the Bianchi II phase space is the attractor for the Bianchi VIII and IX dynamics, see [163. The phase space for the Bianchi II model is given by the Hamiltonian constraint (3.4) together with the condition $N_{1} N_{2}=N_{2} N_{3}=N_{1} N_{3}=0$. This defines a variety consisting of the union of three 2-spheres in $\mathbb{R}^{5}$. The Kasner circle is the intersection of these spheres. The conjecture has been proved in the Bianchi IX case.

Theorem 3.2 ([142]). The Bianchi II variety is the asymptotic attractor for vacuum Bianchi IX.

This result goes a goes a long way towards proving the chaotic nature of the Bianchi IX dynamics.

The questions of curvature blowup and oscillatory approach to the singularity can be studied also in the case of Bianchi class B models. In this case we have curvature blowup except for Bianchi III and V. However, in 

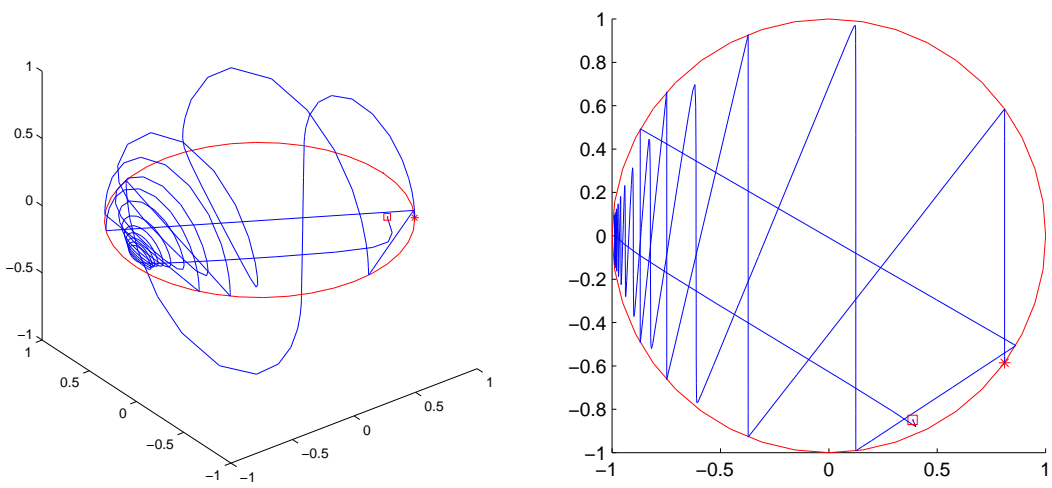

Figure 5. An orbit of the Bianchi $\mathrm{VI}_{-1 / 9}$ system, showing a few bounces. Note that in contrast to the Bianchi IX system, the dynamics shows a combination of several types of bounces.

class B it is only the so-called exceptional Bianchi VI $-1 / 9$ which, based on numerical work and qualitative analysis by Hewitt et. al [87 has an oscillatory singularity. The exceptional Bianchi $\mathrm{VI}_{-1 / 9}$ has the same number of degrees of freedom as the most general class A models, Bianchi VIII and IX. Figure 3 shows an orbit of the Bianchi $\mathrm{VI}_{-1 / 9}$ system.

\section{4. $G_{2}$}

In this section we consider the case when $(\bar{M}, \bar{g})$ is a $3+1$-dimensional, spatially compact, globally hyperbolic, vacuum spacetime, with a 2-dimensional local isometry group $G_{2}$ with the action of $G_{2}$ generated by spacelike Killing fields. By passing to the universal cover, we see that the nondegenerate orbits of the $G_{2}$-action are 2-dimensional homogeneous spaces and hence the induced metric on the orbits must have constant curvature. The isometry group of the sphere $S^{2}$ has no two dimensional subgroup and thus the orbits have geometry $\mathbf{E}^{2}$ or $\mathbf{H}^{2}$.

The special case when the group $\mathrm{U}(1) \times \mathrm{U}(1)$ itself acts on $\bar{M}$ was considered by Gowdy [80, 81]. In this case, it follows that orbits are compact (unless there is an extra Killing field), and $M$ is covered by $T^{3}, S^{3}$ or $S^{1} \times S^{2}$. Suppose $p$ is a fix point for the action of $\mathrm{U}(1) \times \mathrm{U}(1)$ on $M$, then $\mathrm{U}(1) \times \mathrm{U}(1)$ acts by isometries on $T_{p} M$. As the isometry group $\mathrm{SO}(3)$ of $T_{p} M$ does not have a 2-dimensional subgroup, any degenerate orbit of $G$ must be a closed curve.

Let $\bar{M}$ be a bundle over $S^{1} \times \mathbb{R}$ with compact 2-dimensional fiber $F$ and suppose that the orbits of the $G$-action on the universal cover of $\bar{M}$ cover the fibers $F$. If $F$ has geometry $\mathbf{E}^{2}$ it follows that the Killing fields generating the $G$-action commute, and hence it is natural, following Rendall [136], to use the term local $\mathrm{U}(1) \times \mathrm{U}(1)$ symmetry for this situation. 
Space-times with local $\mathrm{U}(1) \times \mathrm{U}(1)$ symmetry have also been considered by Tanimoto [155], who discussed the question of in which case a spacetime with local $\mathrm{U}(1) \times \mathrm{U}(1)$ symmetry can be considered as a dehomogenization of a Bianchi spacetime. It can be seen from the structure of the Bianchi groups that the only $\mathrm{U}(1) \times \mathrm{U}(1)$ symmetric spacetimes which have Bianchi (or Kantowski-Sachs) limits are covered by $T^{3}$ or $S^{3}$, and in the case of $S^{3}$ (Bianchi IX), it is only the Taub metrics that admit a $\mathrm{U}(1) \times \mathrm{U}(1)$ action by isometries. If we consider the case with local $\mathrm{U}(1) \times \mathrm{U}(1)$-symmetric case on the other hand, then all Bianchi models except Bianchi VIII and XI, and in case of Bianchi VIII and IX the Taub metrics, can be viewed as limits of locally $\mathrm{U}(1) \times \mathrm{U}(1)$-symmetric models, and therefore these serve as dehomogenization of the Bianchi models.

For simplicity, we concentrate in the rest of this section only the case of $\mathrm{U}(1) \times \mathrm{U}(1)$ symmetric spacetimes, and assume that the twist constants vanish, i.e. i.e. $\xi_{1} \wedge \xi_{2} \wedge d \xi_{1}=\xi_{1} \wedge \xi_{2} \wedge d \xi_{2}=0$, where $\xi_{1}, \xi_{2}$ are one forms dual to the generators of the $\mathrm{U}(1) \times \mathrm{U}(1)$ action. This is a nontrivial restriction only in case $M \cong T^{3}$, see [81, p. 211]. Such spacetimes are known in the literature as Gowdy spacetimes. We further specialize to the case with Cauchy surface $M \cong T^{3}$

Let $\tau, x$ be coordinates on the $1+1$ dimensional Lorentzian orbit space $(\mathrm{U}(1) \times \mathrm{U}(1)) \backslash \bar{M}$ and let $A(\tau, \theta)$ be the area of the orbit. Gowdy showed that in the non-twisted case, with $M \cong T^{3}$, there are no degenerate orbits, i.e. $A \neq 0$, and further, the level sets of $A$ in $(\mathrm{U}(1) \times \mathrm{U}(1)) \backslash \bar{M}$ are space-like. We may therefore choose coordinates so that $A=4 \pi^{2} e^{-\tau}$, and choose the metric on the orbit as $A h$ where $h=h(\tau, x)$ is a unit determinant metric. $\bar{M}$ is causally incomplete in the direction $\tau \nearrow \infty$, which corresponds to a cosmological singularity.

By construction, the metric on the orbit, $h=h(\tau, x)$ is a unit determinant metric which is constant on each orbit. It therefore represents an element of the Teichmüller space $\mathcal{T}\left(T^{2}\right)$. The space $\mathcal{T}\left(T^{2}\right)$ with the Weil-Peterson metric is isometric to the hyperbolic plane $\mathbf{H}^{2}$. The identification of $\mathcal{T}\left(T^{2}\right)$ with $\mathbf{H}^{2}$ gives a map $u:(\mathrm{U}(1) \times \mathrm{U}(1)) \backslash \bar{M} \rightarrow \mathbf{H}^{2}$. This can be realized concretely for example by using the model for $\mathbf{H}^{2}$ with metric

$$
d P^{2}+e^{2 P} d Q^{2}
$$

and letting $u=(P, Q)$ with

$$
P=\ln \left(h_{11}\right), \quad Q=e^{-P} h_{12} .
$$

This is the parametrization that is used in the numerical work of Berger and collaborators. Thus the Gowdy (as well as the general $\mathrm{U}(1) \times \mathrm{U}(1)$ symmetric) Einstein equations on $M \cong T^{3} \times \mathbb{R}$ can be viewed as equations for the evolution of a loop in $\mathbf{H}^{2}$. The velocity of a point $u(x, \tau)$ in $\mathbf{H}^{2}$ is given by $v_{\mathbf{H}^{2}}(x, \tau)=\sqrt{\left\langle\partial_{\tau} u, \partial_{\tau} u\right\rangle}$. Define the asymptotic velocity $\hat{v}_{\mathbf{H}^{2}}(x)$ by $\hat{v}_{\mathbf{H}^{2}}(x)=\lim _{\tau \rightarrow \infty} v_{\mathbf{H}^{2}}(x, \tau)$, when the limit exists. 
The spacetime metric may then be written in the form ${ }^{5}$

$$
\begin{aligned}
\ell_{0}^{-2} d s^{2}= & e^{-\lambda / 2+\tau / 2}\left(-e^{-2 \tau} d \tau^{2}+d x^{2}\right) \\
& +e^{-\tau}\left[e^{P} d y_{2}^{2}+2 e^{P} Q d y_{2} d y_{3}+\left(e^{P} Q^{2}+e^{-P}\right) d y_{3}^{2}\right]
\end{aligned}
$$

where $\ell_{0}$ is the unit of physical length. Here $(\tau, \theta) \in \mathbb{R} \times S^{1}$ are coordinates on the orbit space, $y^{A}, A=2,3$ are coordinates on the orbit, $0 \leq y^{A} \leq 2 \pi$. The $G$ invariance implies that we can assume all metric components depend on $\tau, \theta$ only. Let $e=d x^{2}+\left(d y^{2}\right)^{2}+\left(d y^{3}\right)^{2}$. The lapse function $N$ satisfies $N=\sqrt{\operatorname{det}(g) / \operatorname{det}(e)}$ and hence the time function $\tau$ is spacetime harmonic, cf. subsection 2.3 .

Let $\eta=-d \tau^{2}+e^{2 \tau} d x^{2}$. The Einstein evolution equations take the form

$$
\eta^{\alpha \beta}\left(\partial_{\alpha \beta} u^{a}+\Gamma_{b c}^{a}(u) \partial_{\alpha} u^{b} \partial_{\beta} u^{c}\right)=0,
$$

where $\Gamma_{b c}^{a}$ are the Christoffel symbols on $\mathbf{H}^{2}$ with the metric (4.1). The system (4.3) is supplemented by a pair of equations for $\lambda$, which are implied by the Einstein constraint equations, and which are used to reconstruct the $3+1$ metric $\bar{g}$.

Equation (4.3) is a semilinear hyperbolic system, which resembles the wave-map equation, (2.8). Energy estimates or light cone estimates prove global existence on $(0, \infty) \times S^{1}$. The wave operator $-\partial_{\tau}^{2}+e^{-2 \tau} \partial_{x}^{2}$ degenerates as $\tau \nearrow \infty$, which corresponds to a singularity in the $3+1$ spacetime $(\bar{M}, \bar{g})$, since the area $e^{-\tau}$ of the orbit tends to zero.

The energy $E=\frac{1}{2} \int_{S^{1}}\left\langle\partial_{\tau} u, \partial_{\tau} u\right\rangle+e^{-2 \tau}\left\langle\partial_{x} u, \partial_{x} u\right\rangle=E_{K}+E_{V}$, where $E_{K}, E_{V}$ are the kinetic and potential energy terms, respectively, satisfies

$$
\partial_{\tau} E=-2 E_{V} .
$$

Therefore $E$ is monotone decreasing, with a rate determined by $E_{V}$. This shows immediately that there is a sequence of times $\left(\tau_{k}\right), \lim _{k \rightarrow \infty} \tau_{k}=$ $\infty$, so that $E_{V}\left(\tau_{k}\right) \rightarrow 0$ as $k \rightarrow \infty$, which indicates that the scale-free variables $\left(e^{-\tau} \partial_{x} P, e^{-\tau} \partial_{x} Q\right)$ become insignificant for the dynamics as $\tau \rightarrow \infty$. This leads to the idea that the Gowdy system behaves asymptotically as a dynamical system in the likewise scale-free variables $\left(\partial_{\tau} P, \partial_{\tau} Q\right)$. This heuristic is, as we shall see, supported by numerical work and some rigorous results.

We now briefly discuss some results and open problems for the Gowdy and $\mathrm{U}(1) \times \mathrm{U}(1)$ symmetric spacetimes. The following result proves Conjecture 3 for this class. The first global existence result for Gowdy spacetimes with topology $T^{3} \times \mathbb{R}$ was due to Moncrief [125], who proved that vacuum Gowdy spacetimes with the stated topology are globally foliated by level sets of the area function, assuming the existence of a compact level set of the area function. This assumption was removed by Chrusćiel [4] who also studied global properties of the area function for Gowdy spacetimes on $S^{3}$

\footnotetext{
${ }^{5}$ We have assumed here that the Killing fields are hypersurface orthogonal, see [4] for the most general form of the $\mathrm{U}(1) \times \mathrm{U}(1)$ symmetric metric on $T^{3} \times \mathbb{R}$ with vanishing twist.
} 
and $S^{2} \times S^{1}$. A class of "nongeneric" metrics still remains to be studied, see 47. The first result concerning global CMC foliations in Gowdy spacetimes was proved by Isenberg and Moncrief [99] for the case of vacuum Gowdy spacetimes. Recently the following was proved.

Theorem 4.1 (Andréasson, Rendall, Weaver 14). Nonflat spatially compact spacetimes with $(\mathrm{U}(1) \times \mathrm{U}(1))$ symmetry and Vlasov matter, are globally foliated by CMC hypersurfaces with mean curvature taking on all values in $(-\infty, 0)$.

It was conjectured by Belinskii, Khalatnikov and Lifschitz [120 that in a generic spacetime with a cosmological singularity, spatial points will decouple as one approaches the singularity, and spatial derivatives become insignificant asymptotically. This leads to the idea that asymptotically near the singularity, the dynamics of the gravitational field should be explained by a family of ODE systems. This is a very rough idea. Yet, as it turns out, this principle appears to hold in the cases we have been able to study. The idea of BKL has been specialized and reformulated by among others Eardley, Liang and Sachs 62] and Isenberg and Moncrief [101, into the notion of asymptotically velocity term dominated (AVTD) singularities. Roughly, an AVTD solution approaches asymptotically, at generic spatial points, the solution to an ODE, the parameters of which depend on the spatial point. In particular, in an AVTD spacetime, locally near a fixed spatial point the spacetime approaches a Kasner limit, with parameters depending on the spatial point. The asymptotic model for an AVTD spacetime necessarily has a non-oscillatory singularity, which excludes generic spacetimes. The precise formulation of the BKL conjecture for generic spacetimes is more subtle, see 161, 13 for discussion of this problem.

The numerical studies referred to above indicate that for a generic Gowdy spacetime, as $\tau \nearrow \infty$, the velocity $v_{\mathbf{H}^{2}}$ is eventually forced to satisfy $0 \leq$ $v_{\mathbf{H}^{2}} \leq 1$, except at isolated $x$-values, even if $v_{\mathbf{H}^{2}}>1$ in some subsets of $S^{1}$ initially, and further that $v_{\mathbf{H}^{2}}$ has a limiting value $\hat{v}_{\mathbf{H}^{2}}(x)$ for each $x$ as one moves toward the singularity.

The numerical solutions exhibit "spikes" at those $x$-values, where $v_{\mathbf{H}^{2}} \geq 1$ asymptotically, cf. figure 6. The very sharp spikes seen (in $Q$ ) are coordinate effects, corresponding to a part of the solution loop approaching the point on the boundary of $\mathbf{H}^{2}$ sent to infinity by the transformation leading to the model with metric (4.1).

Gowdy spacetimes such that $\partial_{x} Q=0$ are called polarized. Equation (4.3) then becomes linear. It was proved by Isenberg and Moncrief 101 that polarized Gowdy spacetimes are AVTD. Numerical studies by Berger et. al., see [26] and references therein, see also [86, 29], support the idea that general Gowdy spacetimes are AVTD. It should be noted that there are polarized Gowdy spacetimes with $v>1$ up to the singularity, but the above mentioned work indicates that this behavior is non-generic. See also 48 for work on the case $v=0$. 


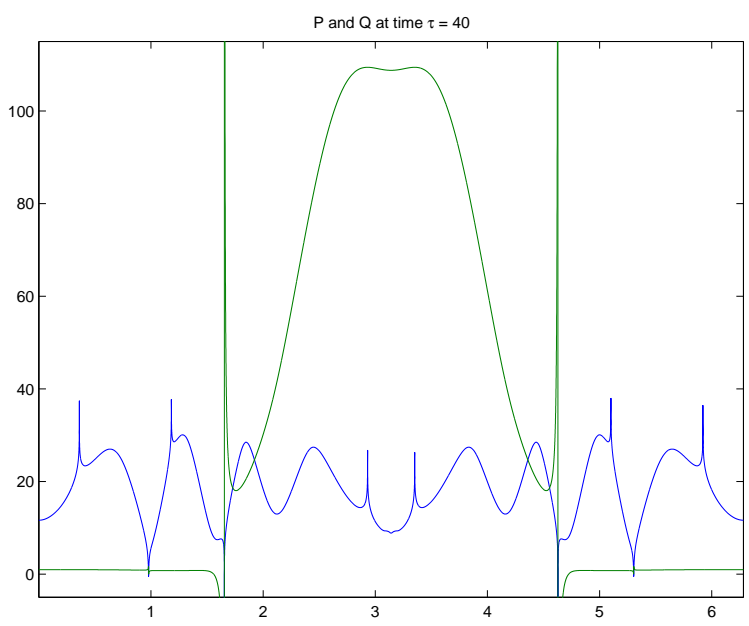

Figure 6. Spikes in $P, Q$. The very sharp spikes (in $Q$ ), so-called "false spikes" are coordinate effects.

The equation (4.3) may, essentially under the restriction $0<v<1$, be written as a Fuchsian system

$$
(t \partial t+E) \mathcal{U}=\mathcal{F}[\mathcal{U}]
$$

where $t=e^{-\tau}$, and hence using a singular version of the Cauchy-Kowalewskaya theorem, cf. Kichenassamy and Rendall [107] and references therein, see also [19], AVTD solutions may be constructed given real analytic "data on the singularity". The solutions constructed are in terms of the time coordinate $\tau$, of the form

$$
\begin{aligned}
& P(\tau, x)=v(x) \tau+\phi(x)+e^{-\epsilon \tau} u(x, \tau) \\
& Q(\tau, x)=q(x)+e^{-2 v(x) \tau}[\psi(x)+w(\tau, x)]
\end{aligned}
$$

where $\epsilon>0,0<v<1$, and $w, u \rightarrow 0$ as $\tau \rightarrow \infty$. The Fuchsian method was generalized for the Gowdy case by Rendall [138] to the $C^{\infty}$ case.

Rendall and Weaver [140] have constructed families of solutions with spikes, starting from a solution with a false spike at $x_{0}$ with $k=\hat{v}_{\mathbf{H}^{2}}\left(x_{0}\right)<1$, by applying an explicit transformation, a new solution with $\hat{v}_{\mathbf{H}^{2}}\left(x_{0}\right)=1+k$. In the new solution $\hat{v}_{\mathbf{H}^{2}}$ has a discontinuity at $x_{0}$ with $\lim _{x \rightarrow x_{0}} \hat{v}_{\mathbf{H}^{2}}(x)=$ $1-k$. By iterating this procedure spikes with arbitrarily high velocity can be constructed. The group formed by composing the transformations used by Rendall and Weaver has been termed the Geroch group 38. Spikes with asymptotic velocity $\hat{v}_{\mathbf{H}^{2}}>2$ correspond to higher order zeros of $\partial_{x} Q$, and are therefore non-generic. Garfinkle and Weaver [77] have studied the dynamics of generic spikes with initally high velocity. Their analysis, based on a combination of heuristic arguments, related to the "method of consistent potentials", see [26] for discussion, and numerical work, shows that 
the velocity of these spikes is driven down into the interval $1<v<2$ by a sequence of bounces, the qualitative features of which can be explained in terms of the relative importance of the terms in the evolution equation.

Recently conditions on initial data (essentially a small energy condition together with restriction on the velocity) have been given by Ringstrom 144, 143, and Chae and Chruściel 38 under which the solution is of the form (4.4). This work shows that AVTD behavior holds on open and dense subsets of $S^{1}$, and makes earlier work 82 on formal expansions for the Gowdy field equations rigorous. The definition of AVTD solution used by Chae and Chrusciel [38, Eq. (3.8-3.9)] is more general than (4.4) used by Ringstrom, allowing more general velocity $v$ but with less precise control on the lower order terms.

In view of the above, it is reasonable to make the following conjecture.

Conjecture 4. Generic vacuum, spatially compact $\mathrm{U}(1) \times \mathrm{U}(1)$-symmetric spacetimes with vanishing twist are AVTD at the singularity, in the complement of an at most countable closed subset $E$ of $S^{1}$. The asymptotic velocity $\hat{v}_{\mathbf{H}^{2}}(x)$ exists for all $x \in S^{1}$ and satisfies $0<\hat{v}(x)<1$ for $x \in S^{1} \backslash E$. $\hat{v}_{\mathbf{H}^{2}}$ is continuous on $S^{1} \backslash E$.

Remark 4.1. (1) This is implicit in Grubišić and Moncrief [82].

(2) Chae and Chruściel 38 have constructed solutions with an asymptotic velocity which is discontinuous on any closed set $F \subset S^{1}$, with nonempty interior. In particular $F$ may have nonzero measure. If the above Conjecture is correct, this behavior is nongeneric.

The question of cosmic censorship for the Gowdy spacetimes may be studied by analyzing the behavior of the Kretschmann scalar $\kappa$ as $\tau \rightarrow \infty$. For the class of polarized Gowdy spacetimes, this was done by Chruściel et. al. 54. It was proved by Kichenassamy and Rendall [107 that for generic AVTD spacetimes constructed using the Fuchsian algorithm, the Kretschmann scalar $\kappa$ blows up at the singularity and hence generically, these spacetimes do not admit extensions, see also the discussion in [82, $\S 3-4]$. The same behavior was shown to hold also for the class of Gowdy spacetimes with asympotic velocity $0<\hat{v}<1$ and bounds on the energy density, see [143, 38]. Further, the Gowdy spikes constructed by Rendall and Weaver also have $\kappa$ blowing up, though at a different rate than nearby points. Therefore a reasonable approach to the SCC in the class of Gowdy spacetimes, is via conjecture 4 .

It is relevant to mention here that the AVTD behavior for Gowdy symmetric spacetimes may be broken by the introduction of suitable matter, cf. [168, where numerical evidence for an oscillatory approach to the singularity is presented, for a locally $\mathrm{U}(1) \times \mathrm{U}(1)$ symmetric spacetime with magnetic field. Similarly, it is expected that scalar field or stiff fluid matter changes the oscillatory behavior of general $\mathrm{U}(1) \times \mathrm{U}(1)$ symmetric models to AVTD. However, even in the presence of a scalar field, one expects to see spikes forming. 


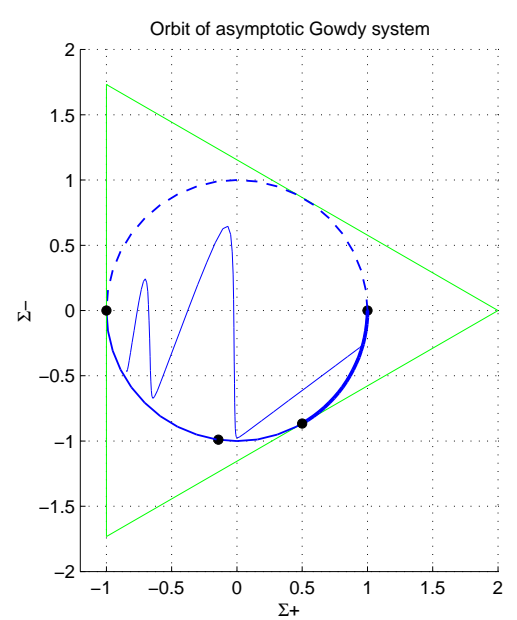

Figure

7. Projection

in Kasner plane of an orbit of the asymptotic Gowdy system (4.54.6).

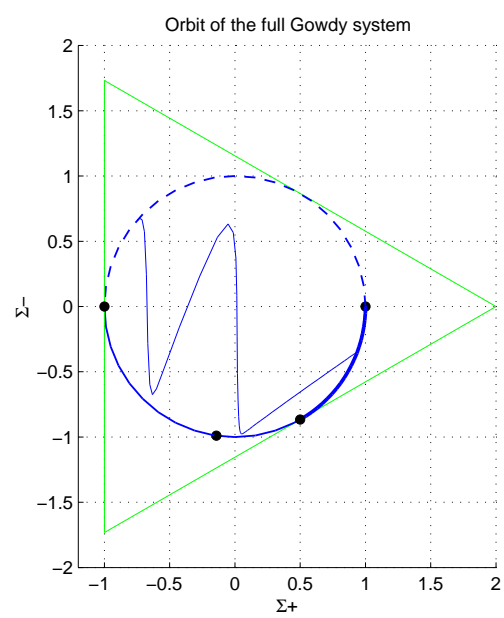

Figure

8. Projection in Kasner plane of the orbit of a point of the full Gowdy system.

A systematic way of deriving the system of ODE's governing the asymptotic behavior of the Gowdy spacetimes is to write (4.3) in first order form, using the scale invariant operators $\partial_{\tau}, e^{-\tau} \partial_{x}$, and then cancelling the terms involving $e^{-\tau} \partial_{x}$. Following this procedure and working in terms of $H$ normalized orthonormal frame variables adapted to the $\mathrm{U}(1) \times \mathrm{U}(1)$ orbits, constructed in a completely analogous way to those discussed in section 3 gives, after cancelling the terms corresponding to $e^{-\tau} \partial_{x}$, the system

$$
\begin{aligned}
& \mathcal{D}_{0} \Sigma_{+}=(q-2)\left(1+\Sigma_{+}\right) \\
& \mathcal{D}_{0} \Sigma_{-}=(q-2) \Sigma_{-}-2 \sqrt{3} N_{-}^{2}+2 \sqrt{3} \Sigma_{\times}^{2} \\
& \mathcal{D}_{0} N_{\times}=\left(q+2 \Sigma_{+}\right) N_{\times} \\
& \mathcal{D}_{0} \Sigma_{\times}=\left(q-2-2 \sqrt{3} \Sigma_{-}\right) \Sigma_{\times}-2 \sqrt{3} N_{\times} N_{-} \\
& \mathcal{D}_{0} N_{-}=\left(q+2 \Sigma_{+}+2 \sqrt{3} \Sigma_{-}\right) N_{-}+2 \sqrt{3} \Sigma_{\times} N_{\times} .
\end{aligned}
$$

where $\mathcal{D}_{0}=\left(1+\Sigma_{+}\right) \partial_{\tau}, q=2\left(\Sigma_{+}^{2}+\Sigma_{-}^{2}+\Sigma_{\times}^{2}\right)$, subject to the constraint equation

$$
1=\Sigma_{+}^{2}+\Sigma_{-}^{2}+\Sigma_{\times}^{2}+N_{\times}^{2}+N_{-}^{2}
$$

See 13 for discussion of the system (4.5) 4.6). This system contains the spatially homogenous Bianchi class A models $\mathrm{I}-\mathrm{VII}_{0}$, but represented in terms of a non-Fermi-propagated frame.

The asymptotic behavior of the system (4.5 4.6) in the direction towards the singularity is easily analyzed, and one shows that all solutions of the 
system have limit points on the Kasner half-circle $\Sigma_{+}^{2}+\Sigma_{-}^{2}=1$. Similar to the standard Bianchi case, the Kasner circle consists of fixed points. For the Bianchi types II-VII 0 , only part of the Kasner circle consists of stable fixed points. In the present case, only the arc with $\Sigma_{-} \leq 0, \Sigma_{+}>\frac{1}{2}$ is stable, and a generic orbit of (4.5) ends on the stable arc. The stable arc corresponds to orbits with asymptotic velocity $0<\hat{v}<1$. See figure 7 for an example of an orbit of the system (4.54 4.6).

According to the BKL proposal, a generic solution $u(t, x)$ of the full Gowdy system will have the property that it will asymptotically approach a solution of the Gowdy AVTD system (4.544.6). That this is indeed the case is indicated by figure 8, which shows the projection in the Kasner plane of the orbit of one point for the full Gowdy system. We see in the figure the orbit of a point near a spike point. The points marked on the Kasner circle are (from the left) the points with velocity $v=\infty, 2,1,0$. The stable arc on the Kasner circle is plotted with a heavy line. It should be noted that the last part of the orbit which ends on the stable Kasner arc is approximately a Bianchi II orbit, with initial velocity in the interval $(1,2)$. This is the generic behavior, also close to spike points, see [13] for more details.

A class of AVTD polarized Gowdy spacetimes with non-vanishing twist has been constructed by Isenberg and Kichenassamy [96] using the Fuchsian algorithm. On the other hand for general non-polarised twisted $\mathrm{U}(1) \times \mathrm{U}(1)$ symmetric spacetimes, work of Berger et al. 31] supports the conjecture that these spacetimes show oscillating behavior as one approaches the singularity. This idea is also supported by the fact that the only Bianchi model with $\mathrm{U}(1) \times \mathrm{U}(1)$ symmetry and non-vanishing twist constants is the exceptional Bianchi VI $-1 / 9$ which has been shown heuristically by Hewitt et al. [87] to have oscillatory behavior at the singularity.

\section{5. $\mathrm{U}(1)$}

The U(1) symmetric vacuum $3+1$ Einstein equations is an important case which is of intermediate difficulty between the full $3+1$ Einstein equations and the highly symmetric Gowdy equations. In the presence of a hypersurface orthogonal space-like Killing field, the Einstein equations reduce to $2+1$ gravity coupled to wave map matter, the field equations and their reductions have been derived in [127, 126, 128, see also 37. Choquet-Bruhat and Moncrief have proved that the Cauchy problem for the $\mathrm{U}(1)$ problem is well-posed in $H^{2}$, see [42, 39].

In the above quoted papers, the spacetime is assumed to be a $\mathrm{U}(1)$ bundle over a spatially compact $2+1$ spacetime. The case of local U(1) symmetry does not appear to have been considered in connection with the Einstein equations, see however 131 for information about 3-manifolds with local $\mathrm{U}(1)$ action.

It is also possible to study the case when the reduced space is asymptotically flat. This case has been considered in work by Ashtekar and others, 
[17, 15, see also [16. In these papers an analogue of the ADM mass at spatial infinity is introduced. It is proved that it is nonnegative and bounded from above. It is interesting to study the consequences of the presence of this conserved quantity for the $2+1$ dimensional Einstein-matter system given by the $\mathrm{U}(1)$ problem, as one expects that it gives a stronger bound on the fields than in the $3+1$ case. This appears to be a natural setting for a small data version of the $\mathrm{U}(1)$ problem.

In the following, we will consider the spatially compact case. Let $(\bar{M}, \bar{g})$ be a $3+1$ dimensional spacetime, assume $\bar{M} \cong B \times \mathbb{R}$, with $\pi: B \rightarrow \Sigma$ a principal U(1) bundle, $\Sigma$ a compact surface. Further assume the group U(1) acts by isometries on $(\bar{M}, \bar{g})$ with the action generated by the Killing field $J$, which we assume to be space-like, $\langle J, J\rangle>0$.

Let the function $\lambda$ on $\Sigma \times \mathbb{R}$ be defined by $\pi^{*} \lambda=\frac{1}{2} \log (\langle J, J\rangle)$, and let $\theta=e^{-2 \pi^{*} \lambda} J$. Then we can write the spacetime metric $\bar{g}$ in the form

$$
\bar{g}=\pi^{*}\left(e^{-2 \lambda} g\right)+e^{2 \pi^{*} \lambda} \theta \otimes \theta,
$$

where $g$ is a Lorentzian metric on $\mathbb{R} \times \Sigma$.

Introduce a frame $e_{\alpha}, \alpha=0,1,2,3$ with $e_{3}=e^{-\lambda} J$, and let the indices $a, b, c, \cdots=0,1,2$. We may without loss of generality assume that $\left[J, e_{a}\right]=0$. We have

$$
d J_{\alpha \beta}=\Theta_{\alpha \beta}+2\left(e_{\alpha}(\lambda) J_{\beta}-e_{\beta}(\lambda) J_{\alpha}\right),
$$

where $\Theta_{\alpha \beta} J^{\beta}=0$. It follows that $\Theta_{\alpha \beta}=\pi^{*}\left(e^{2 \lambda} F_{\alpha \beta}\right)$ where $F_{\alpha \beta}$ is a 2-form on $\mathbb{R} \times \Sigma$. If $F_{\alpha \beta}=0$, then $J$ is hypersurface orthogonal.

To avoid cluttering up the notation we will in the following make no distinction between fields on the orbit space $\mathbb{R} \times B$ and their pullbacks by $\pi$.

The components of the $3+1$ Ricci tensor are

$$
\begin{aligned}
& \bar{R}_{a b}=R_{a b}-2 \nabla_{a} \lambda \nabla_{b} \lambda+\nabla_{c} \nabla^{c} \lambda g_{a b}-\frac{1}{2} e^{4 \lambda} F_{a c} F_{a}{ }^{c}, \\
& \bar{R}_{a 3}=\frac{1}{2} e^{-\lambda} \nabla_{c}\left(e^{4 \lambda} F_{a}{ }^{c}\right), \\
& \bar{R}_{33}=e^{2 \lambda}\left[-g^{a b} \nabla_{a} \nabla_{b} \lambda+\frac{1}{4} e^{4 \lambda} F_{a b} F^{a b}\right]
\end{aligned}
$$

Let the one-form $E$ on $\mathbb{R} \times \Sigma$ be given by $E=-\star_{g}\left(e^{4 \lambda} F\right)$. One of the Einstein equations (5.1) implies that $d E=0$.

Now the Einstein vacuum equations $\bar{R}_{\alpha \beta}=0$ imply the system

$$
\begin{aligned}
R_{a b} & =\frac{1}{2}\left(4 \nabla_{a} \lambda \nabla_{b} \lambda+e^{-4 \lambda} E_{a} E_{b}\right), \\
\nabla^{a} \nabla_{a} \lambda+\frac{1}{2} e^{-4 \lambda} E_{a} E_{b} g^{a b} & =0, \\
\nabla^{a} \nabla_{a} \omega-4 \nabla^{a} \lambda E_{a} & =0 .
\end{aligned}
$$

\footnotetext{
${ }^{6}$ In [41, a frame $d x^{a}, \theta^{3}$ is used, where $\theta_{\alpha}^{3}=e^{-2 \lambda} J_{\alpha}=e^{-\lambda} e_{\alpha}^{3}$. Furthermore, their $F_{\alpha \beta}=d \theta_{\alpha \beta}=e^{-2 \lambda} \Theta_{\alpha \beta}$.
} 
When $E_{a}=\nabla_{a} \omega$, we recognise (5.2) as the $2+1$ dimensional Einstein equations with wave map matter, for the wave map with components $(\lambda, \omega)$ with target hyperbolic space $\mathbf{H}^{2}$ with the constant curvature metric

$$
2 d \lambda^{2}+\frac{1}{2} e^{-4 \lambda} d \omega^{2} .
$$

See subsection 2.4 for some discussion of wave map equations.

We now specialise even further to the polarized case $E_{a}=0$. This corresponds to assuming that the bundle $\pi: B \rightarrow \Sigma$ is trivial, and that the vector field $J$ is hypersurface orthogonal. Then $\omega$ is constant and the equations (5.2) become

$$
\begin{aligned}
R_{a b} & =\nabla_{a} \lambda \nabla_{b} \lambda \\
\nabla^{a} \nabla_{a} \lambda & =0
\end{aligned}
$$

which is precisely the $2+1$ Einstein equations coupled to a massless scalar field.

In the 2-dimensional case, the operator $k \rightarrow \operatorname{div} k$ is elliptic on symmetric 2 -tensors with vanishing trace. Further, by the uniformization theorem, a compact 2-dimensional Riemannian manifold $(\Sigma, h)$ is conformal to $(\Sigma,[h])$ where $[h]$ is a representative of the conformal class of $g$, i.e. a constant curvature metric. Therefore working in spatial harmonic gauge with respect to $(\Sigma,[h])$ (i.e. conformal spatial harmonic gauge) and CMC time gauge, the constraint equations form an elliptic system for $\left(h_{i j}, k_{i j}\right)$. We get a representation of $\left(h_{i j}, k_{i j}\right)$ in terms of $\left([h], \phi, k^{\mathrm{TT}}, Y\right)$ where $[h]$ is the conformal class of $h$, corresponding to a point in Teichmüller space, $\phi$ a conformal factor determined by the Hamiltonian constraint equation (a nonlinear elliptic system for $\phi), k^{\mathrm{TT}}$ a trace-free, divergence free 2 -tensor on $(\Sigma,[h])$, corresponding to a quadratic differential, and finally, $Y$ is a vector field determined from the momentum constraint equation. Note that $\left([h], k^{\mathrm{TT}}\right)$ represents a point in $T^{*} \mathcal{T}(\Sigma)$, the cotangent bundle of the Teichmüller space of $\Sigma$.

Due to the ellipticity of the constraint equations in the $2+1$ dimensional case, in the gauge as described above, it is possible to eliminate the Einstein equation (5.2a) from the system (5.2) and instead solve the elliptichyperbolic system consisting of the hyperbolic system (5.2b] [5.2c), coupled to a nonlinear system which determines components of the spacetime metric $\bar{g}$ in terms of the data via the constraint and gauge fixing equations, as well as an ODE system determining the evolution of the Teichmuller degrees of freedom of the metric on $\Sigma$. In the special case $\Sigma=S^{2}$, Teichmuller space is a point, and further $H^{1}\left(S^{2}\right)=0$, which means that $E=d \omega$. Therefore ignoring problems with gauge fixing, the reduced $U(1)$ system would consist of the wave equation coupled to the system which determines the components of $\bar{g}$.

A special case of the polarized $\mathrm{U}(1)$ equations is given by setting $\lambda \equiv$ constant. Then the field equations (5.3) are just

$$
R_{a b}=0,
$$


the $2+1$ dimensional vacuum equations. In this case, the spacetime is a 3 -dimensional Lorentzian space-form. The dynamics of $2+1$ dimensional vacuum gravity has been studied by Andersson, Moncrief and Tromba 11 who proved global existence in CMC time for $2+1$ dimensional vacuum spacetimes, with cosmological constant, containing at least one CMC hypersurface. This proves Conjecture 3 for the class of $2+1$ dimensional vacuum spacetimes.

Andersson and Rendall 12 showed that the $3+1$ dimensional Einstein scalar field system can be formulated as a Fuchsian system. Using similar techniques, Damour et al. 61, §4] have shown that the polarized U(1) equations may be formulated as a Fuchsian system, and therefore AVTD solutions may be constructed using a singular version of the Cauchy-Kowalewskaya theorem, as was done by Kichenassamy and Rendall [107 for the Gowdy case. See also Isenberg and Moncrief [102] which deals with the "halfpolarized" U(1) system in addition to the polarized case. This supports the following conjecture.

Conjecture 5. Generic polarized $\mathrm{U}(1)$ spacetimes are AVTD at the singularity.

Remark 5.1. Conjecture 5 was essentially stated by Grubišić and Moncrief 83. This is supported by numerical work of Berger and Moncrief [33. Similarly to the case of Gowdy spacetimes, see Conjecture 4. one expects that "spiky features" will form at the singularity, and hence the AVTD property of the $\mathrm{U}(1)$ symmetric spacetimes should be understood to be generic also in the spatial sense.

It seems reasonable to expect that polarized $U(1)$ spacetimes which are AVTD at the singularity have a strong curvature singularity generically, and therefore that proving the AVTD conjecture for polarized $\mathrm{U}(1)$ would be a big step towards proving SCC for this class.

In contrast to polarized $\mathrm{U}(1)$, the generic $\mathrm{U}(1)$ spacetimes have sufficiently many degrees of freedom that one expects them to satisfy the BKL picture of an oscillatory approach to the singularity, as is also expected in the fully $3+1$ dimensional case, cf. the remarks in section 7 . This is supported by the numerical evidence so far, see 32 .

In the expanding direction, on the other hand, a small data semi-global existence result holds [42, 39, similar to the one for the full $3+1$ dimensional case discussed below in section [6. In this case, the notion of "small data" is taken to be data close to data for a background spacetime with space-like slice $M=\Sigma \times S^{1}$ where $\Sigma$ is a Riemann surface of genus $>2$ with metric $\sigma$ of scalar curvature -1 , and $M$ has the product metric. In this case, the background spacetimes are products of flat spacetimes as in Example 2.5 with $n=2$, with the circle (such spacetimes are of type Bianchi III). The proof uses energy estimates for a second order energy, which controls the $H^{2} \times H^{1}$ norm of the data, for small data. The energy expression used is a combination of several terms. The first order part is $\frac{\tau^{2}}{4} \operatorname{Vol}_{g}(\Sigma)+2 \pi \chi(\Sigma)$, 
which via the constraint equations may be related to the wave map energy. The second order term is defined in terms of the $L^{2}$ norm of the Laplacian of the wave map field, and the gradient of its time derivative. In order to use this method, one of the technical problems one has to deal with is controlling the spectral gap of the Laplacian. This is done by keeping control of the Teichmuller parameters during the evolution. For technical reasons, the assumption is made that the spectral gap $\Lambda_{0}$ of the Laplacian for the inital metric satisfies $\Lambda_{0}>1 / 8$. The proof shows that the Teichmuller parameter $\sigma(t)$ converges to a point in the interior of Teichmuller space and that the spacetime geometry converges in the expanding direction to one of the model spacetimes described above.

\section{6. $3+1$}

In the $3+1$ dimensional case with no symmetries, the only known facts on the global properties of spacetimes are Lorentzian geometry results such as the Hawking-Penrose singularity theorems [132] and the Lorentzian splitting theorem of Galloway 75, see 24 for a survey of Lorentzian geometry.

Here we are interested in results relevant to the SCC, Conjecture 1 and the CMC conjecture, Conjecture 3, i.e. results about the global behavior of solutions to the Cauchy problem for the evolution Einstein equations, in some suitable gauge. The Cauchy problem for the Einstein evolution equations has been discussed in section 2.2 ,

With this limitation there are essentially 3 types of results known and all of these are small data results. The results are those of Friedrich on the "hyperboloidal Cauchy problem", of Christodoulou and Klainerman on the nonlinear stability of Minkowski space (generalized by Klainerman and Nicolò [11] to exterior domains) and the work of Andersson and Moncrief 9, on global existence to the future for data close to the data for certain spatially compact flat $\kappa=-1$ (local) FRW spacetimes, again a nonlinear stability result. We will briefly discuss the main features of these results.

The causal structure of a Lorentz space is a conformal invariant. This leads to the notion that the asymptotic behavior of spacetimes can be studied using conformal compactifications or blowup. The notion of isolated system in general relativity has been formalized by Penrose in terms of regularity properties of the boundary of a conformally related spacetime $(\tilde{M}, \tilde{g})$, with null boundary $\mathcal{J}$, such that $\tilde{M}$ is a completion of $\bar{M}$, and $\bar{g}=\Phi^{*}\left(\Omega^{-2} \tilde{g}\right)$, where $\Omega \in C^{\infty}(\bar{M})$ is a conformal factor, $\Phi: \bar{M} \rightarrow \tilde{M}$ is a diffeomorphism of $\bar{M}$ to the interior of $\tilde{M}$. Given assumptions on the geometry of $(\tilde{M}, \tilde{g})$ at $\mathcal{J}$, Penrose proved using the Bianchi identities that the components of the Weyl tensor of $(\bar{M}, \bar{g})$ decay at physically reasonable rates.

Friedrich derived a first order symmetric hyperbolic system from the Einstein equations, the "regular conformal field equations". This system includes among its unknowns, components of the Weyl tensor, the conformal factor $\Omega$ and quantities derived from the conformally rescaled metric $\tilde{g}$. This 
system has the property that under the Penrose regularity conditions at $\mathcal{J}$, the solution can be extended across J. The fact that the regular conformal field equations gives a well posed evolution equation in the conformally compactified picture enabled Friedrich to prove small data global existence results by using the stability theorem for quasi-linear hyperbolic equations.

In 69, Theorem 3.5] Friedrich proved global existence to the future for data $(M, g, k)$ close to the standard data on a hyperboloid in Minkowski space, satisfying asymptotic regularity conditions compatible with a Penrose type compactification (the hyperboloidal initial value problem). This was later generalized to Maxwell and Yang-Mills matter in [70]. Initial data for the hyperboloidal initial value problem were first constructed by Andersson, Chruściel and Friedrich [6], see also [4], [5].

The result of Friedrich is a semi-global existence result, in the sense that the maximal vacuum Cauchy development $D(M)$ of the data $(M, g, k)$ is proved to be geodesically complete and therefore inextendible to the future, but not to the past. In fact typically $D(M)$ will be extendible to the past and $(M, g, k)$ may be thought of as a partial Cauchy surface in a larger maximal globally hyperbolic spacetime $(\bar{M}, \bar{g})$, cf. figure 9. In view of the inextendibility to the future of $D(M)$, the result of Friedrich may be viewed as supporting the cosmic censorship conjecture. In the case of the Einstein equations with positive cosmological constant, the method of Friedrich yields a global existence result for data close to the standard data on $M=S^{3}$ in deSitter space, cf. [69, Theorem 3.3].

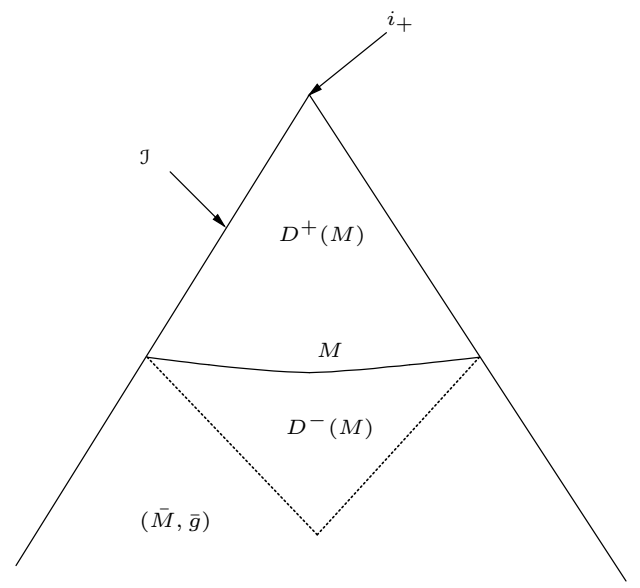

Figure 9. The semi-global existence theorem of H. Friedrich

The first true small data global existence result for the vacuum Einstein equations, was proved by D. Christodoulou and S. Klainerman [46]. They proved that for data $(M, g, k)$ sufficiently close to standard data on a hyperplane in Minkowski space, with appropriate decay at spatial infinity, the $\operatorname{MVCD}(\bar{M}, \bar{g})$ is geodesically complete and therefore inextendible, cf. figure 
10. The Christodoulou-Klainerman global existence theorem therefore supports the cosmic censorship conjecture. Klainerman and Nicolò [110, 111]

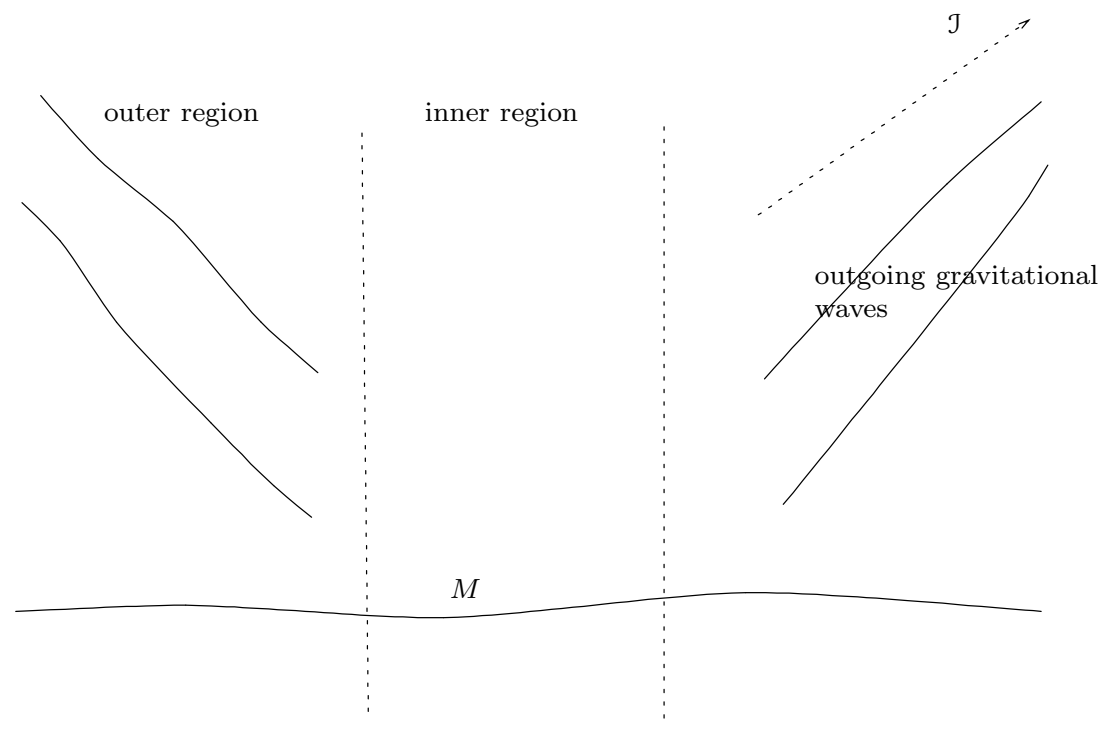

Figure 10. The global existence theorem of D. Christodoulou and S. Klainerman

have proved a global existence result for data $(M, g, k)$ which are close to standard flat Minkowski space data on an exterior region $M \backslash K$, where $K \subset M$ is compact so that $M \backslash K \cong \mathbb{R}^{3} \backslash$ Ball, and with weaker asymptotic conditions compared to the Christodoulou-Klainerman theorem. The result of Klainerman and Nicoló states that for a vacuum data set $(M, g, k)$ which is sufficiently close to the standard flat Minkowski data on $M \backslash K$, the outgoing null geodesics in the causal exterior region $D(M \backslash K)$ are complete and $D(M \backslash K)$ is covered by a double null foliation, with precise control over the asymptotics. It should be noted that the KN theorem therefore covers a more general class of spacetimes than the Christodoulou-Klainerman theorem and is not strictly a small data result. In particular, the maximal Cauchy vacuum development of $(M, g, k)$ may be singular for data satisfying the assumptions of the KN theorem, cf. figure 11] If the smallness assumption is extended also to the interior region, the Christodoulou-Klainerman theorem is recovered. The Einstein equations are quadratic in first derivatives, and therefore in $3+1$ dimensions, one needs something like a null condition in order to get sufficient decay for a global existence argument. The Einstein equations are not known to satisfy a null condition. However, by a detailed construction of approximate Killing fields and approximate conformal Killing fields, Christodoulou and Klainerman are able to control the behavior of components of Bel-Robinson tensors constructed from the Weyl tensor and its derivatives, and close a bootstrap argument which gives global existence for sufficiently small data. As part of this argument, it is 


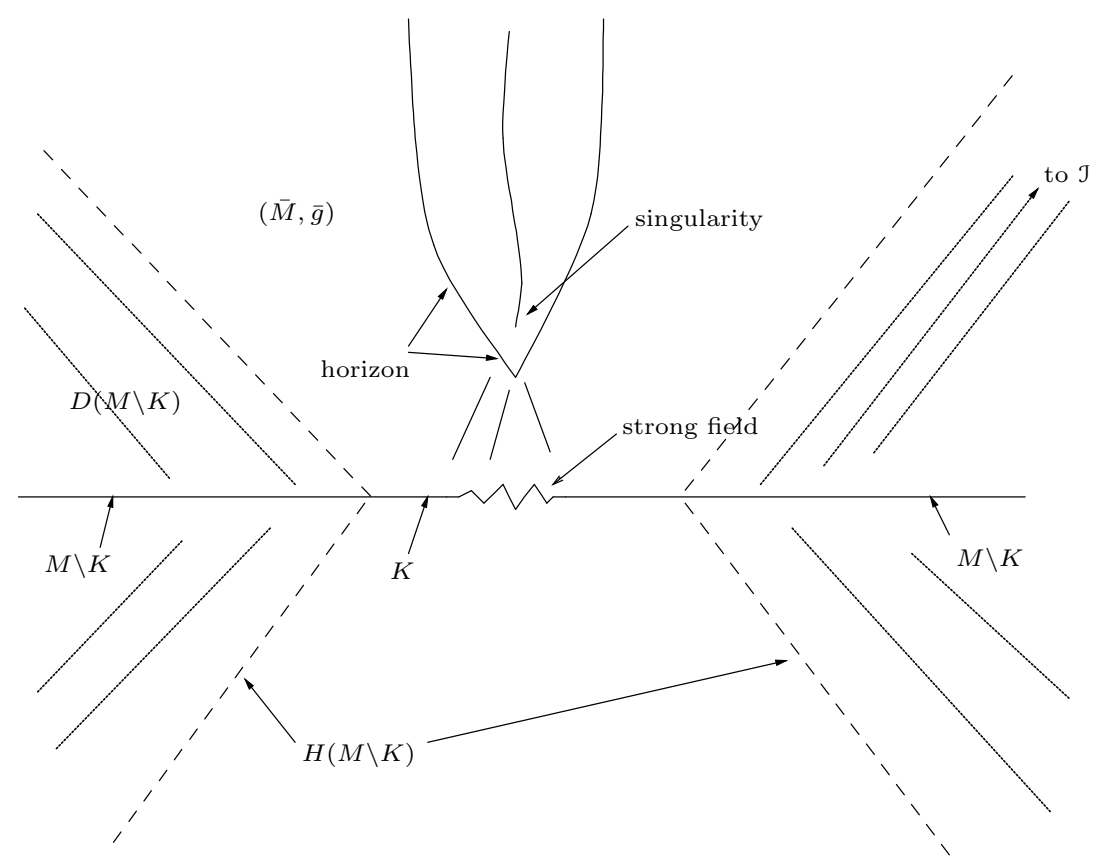

FiguRE 11. The exterior global existence theorem of S. Klainerman and F. Nicoló. The figure illustrates a situation which is covered by this theorem, with a singularity forming due to a strong gravitational field in the interior region $I(K)$ while the exterior region $D(M \backslash K)$ has complete outgoing null geodesics reaching $\mathcal{J}$.

necessary to get detailed control over the asymptotic behavior of light cones. Christodoulou and Klainerman also study the asymptotic behavior of components of the Weyl tensor and are able to prove that some, but not all of these have the decay implied by the Penrose conditions on $\mathcal{J}$.

The question about existence of vacuum asymptotically Minkowskian spacetimes with regular conformal completion, in the sense of Penrose, has been open for a long time. Recently, Delay and Chruściel [51] were able to use a gluing construction of Corvino and Schoen [59 to construct vacuum spacetimes which are exactly Schwarzschild near spatial infinity, and which are close enough to Minkowski space, so that the global existence result of Friedrich applies to show existence of a global J. Further, Klainerman and Nicoló 112 have been able to show that a class of asympotically Euclidean initial data with suitable decay at infinity has a Cauchy development where the null components of the Weyl tensor have decay corresponding to the peeling conditions of Penrose.

The work in [6] and 4] shows that generic hyperboloidal data do not satisfy the required regularity at the conformal boundary, which therefore may be viewed as an indication that Penrose regularity at $\mathcal{J}$ is non-generic. 
It is still an open question what general conditions on initial data on an asymptotically flat Cauchy surface give a Cauchy development with regular conformal completion. H. Friedrich has been developing a programme which approaches this problem by analyzing the conformal structure at spatial infinite in detail, see [60 for references on this, and see also the paper [162] by Valiente-Kroon, which points out some new obstructions to regularity.

In contrast to the results of Friedrich and Christodoulou-KlainermanNicoló, the semi-global existence result of Andersson and Moncrief [9] deals with spatially compact vacuum spacetimes.

Let $(M, \gamma)$ be a compact hyperbolic 3-manifold with metric $\gamma$ of sectional curvature -1 . Then the spacetime $\bar{M}=M \times \mathbb{R}$ with metric $\bar{\gamma}=-d t^{2}+t^{2} \gamma$ is a $\kappa=-1$ (local) FRW spacetime. A flat spacetime metric on $\bar{M}$ defines a geometric structure with group $S O(3,1) \ltimes \mathbb{R}^{4}$. The moduli space of flat spacetimes with topology $M \times \mathbb{R}$ has the same dimension as the moduli space of flat conformal structures on $M$, see [3]. We say that $M$ is rigid if the dimension of the moduli space of flat conformal structures on $M$ is zero. This is equivalent to the condition that there are no nonzero traceless Codazzi tensors on $(M, \gamma)$. Kapovich [106] has constructed examples of rigid compact hyperbolic 3-manifolds.

The global existence theorem proved in [9] states that if $M$ is a rigid manifold of hyperbolic type, then for a vacuum data set $(M, g, k)$, sufficiently close to the standard data in a spatially compact $\kappa=-1$ (local) FRW spacetime, the $\operatorname{MVCD}(\bar{M}, \bar{g})$ is causally geodesically complete in the expanding direction. It is a consequence of the singularity theorem of Hawking and Penrose that $(\bar{M}, \bar{g})$ is singular, i.e. geodesics in the collapsing direction are incomplete, cf. figure 12

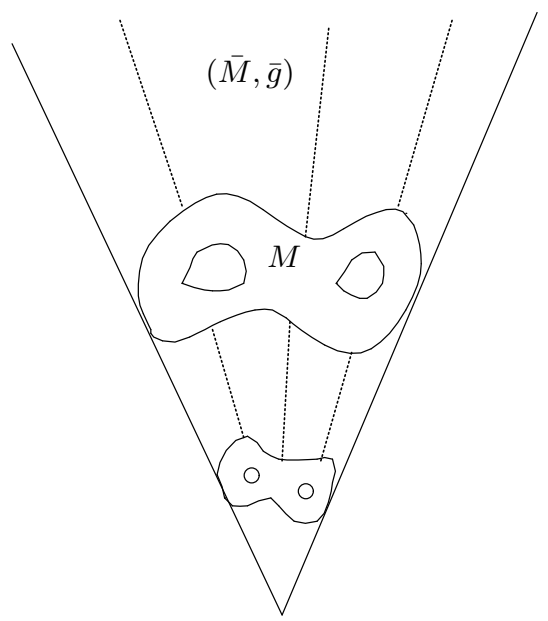

Figure 12. The semi-global existence theorem of L. Andersson and V. Moncrief. 
The proof uses local well posedness and a continuation principle for the Einstein equations in constant mean curvature and spatial harmonic coordinates (CMCSH) gauge, cf. subsection 2.3. together with energy estimates for a first order Bel-Robinson type energy, defined in terms of the Weyl tensor and its covariant derivative w.r.t. $T$. Let $W_{a b c d}$ be the Weyl tensor and let ${ }^{*} W_{a b c d}$ be its dual. In a vacuum spacetime $W_{a b c d}=R_{a b c d}$. Define $E_{i j}=W_{i T j T}$ and $B_{i j}={ }^{*} W_{i T j T}$. Then $E_{i j}$ and $B_{i j}$ are the electric and magnetic parts of the Weyl tensor. These fields are traceless and symmetric and satisfy a Maxwell-like system. A computation shows that the zeroth order Bel-Robinson energy

$$
\mathcal{Q}=\int|E|^{2}+|B|^{2}
$$

(which measures second derivatives of $g$ in $L^{2}$ ), satisfies the equation

$$
\begin{aligned}
\partial_{t}= & -3 \int_{M_{t}} N\left[(E \times E) \cdot k+(B \times B) \cdot k-\frac{1}{3}\left(|E|^{2}+|B|^{2}\right) \operatorname{tr} k\right. \\
& \left.-2 N^{-1} \nabla^{i} N(E \wedge B)_{i}\right] d \mu_{M_{t}}
\end{aligned}
$$

where for symmetric traceless two-tensors $A, B$,

$$
(A \times B)_{i j}=A_{i}{ }^{m} B_{m j}+A_{j}^{m} B_{m i}-\frac{2}{3} A^{m n} B_{m n} g_{i j}
$$

and

$$
(A \wedge B)_{i}=\epsilon_{i}^{m n} A_{m}^{k} B_{k n},
$$

Combining $\mathcal{Q}$ with the a term defined analogously in terms of $\bar{\nabla}_{T} W_{a b c d}$ and extracting a scale invariant quantity, leads to an energy $\mathcal{E}$, which bounds the Cauchy data in $H^{3} \times H^{2}$ for small data. This energy is shown to satisfy an energy inequality of the form

$$
\partial_{t} \mathcal{E} \leq-2 \mathcal{E}-C \mathcal{E}^{3 / 2}
$$

Using this one may show that if $\mathcal{E}$ is small initially, then $\mathcal{E}$ will decrease, as long as local existence holds. A continuation principle for the Einstein equations in $H^{3} \times H^{2}$ [10] completes the proof of global existence for small data.

\section{Concluding Remarks}

The results and numerical studies so far can be argued to fit with the so called BKL picture of cosmological singularities, which states that the generic singularity should be space-like, local and oscillatory, see [28] for a review. Roughly, one expects that the "points on the singularity" are causally separated and that locally at the singularity, the dynamics undergoes a chaotic sequence of curvature driven "bounces" interspersed with relatively uneventful "coasting" epochs, with Kasner like dynamics (i.e. the metric is, locally in space, approximately of the form given in (3.6)). The locality at the singularity, is easily checked for the nonvacuum FriedmanRobertson-Walker models of the standard model of cosmology, and is the 
cause of the so-called "horizon problem" in cosmology. Locality at the singularity can be proved for Gowdy and polarized U(1).

The picture with bounces and coasting is to a large extent inspired by the Mixmaster model (Bianchi IX, cf. section 3) which is known to have an oscillatory singularity, cf. Theorem 3.1. and the above scenario may therefore be described by the slogan "generic singularities are spacelike and oscillatory". By an abuse of language we may say that the oscillatory behavior is of "mixmaster type".

An approach to understanding the asymptotic dynamics near singularities is to attempt to extract, in a systematic way, a dynamical system governing the asymptotic behavior of the Einstein equations. A promising approach is based on an analysis of the evolution equations written in Hubble-normalized scale-free orthonormal frame variables along the lines discussed for the Gowdy system in section 4 . The general situation has been discussed in [161. In this setting the Einstein evolution equations appear in first order form, and the natural version of the BKL conjecture is that $\partial_{1} U \rightarrow 0$ in the direction of the singularity, where $\partial_{1}$ is a scalefree first order operator analogous to the operator $e^{-\tau} \partial_{x}$ for the Gowdy system, and $U$ denotes the scale free frame variables.

If this picture is correct, the asymptotic behavior near the singularity can be understood by analyzing the asymptotic behavior of orbits of the system of ODE's that one gets by cancelling the terms containing $\partial_{\mathbf{1}}$ in the first order form of the Einstein evolution equations. The possibilities that arise have been analyzed in [161]. It is now important to understand whether or not this version of the BKL conjecture is correct by doing numerical experiments. No complete results in this direction have been obtained so far.

The mixmaster type behavior may be prevented by the presence of certain types of matter such as a scalar field (stiff fluid). In fact work by Andersson and Rendall 12 , shows that the $3+1$ dimensional Einstein-scalar field equations may be formulated as a Fuchsian system near the singularity, and AVTD solutions may be constructed using a singular version of the CauchyKowalevskaya theorem. For the polarized U(1) case, the reduced system is $2+1$ dimensional Einstein-scalar field equations. The Fuchsian method has been applied to this special case by Isenberg and Moncrief 102. Damour et al. 61 have given a rather complete study of the application of the Fuchsian method to spacetimes of general dimension with matter.

It appears likely that some aspect of the picture sketched above will be relevant for the final analysis of the large data, global behavior of vacuum spacetimes.

\section{Appendix A. Basic Causality concepts}

Here we introduce the basic causality concepts, see [165, Chapter 8], 24, 84, 132 for details. 
A compact manifold $\bar{M}$ admits a Lorentz metric if and only if its Euler characteristic $\chi(\bar{M})$ vanishes, while all noncompact manifolds admit Lorentz metrics. A vector $V \in T \bar{M}$ is called space-like, null or time-like if $\bar{g}(V, V)>$ $0,=0$ or $<0$ respectively. $V$ is called causal if it is null or time-like. A $C^{1}$ curve $c \subset \bar{M}$ is called time-like (causal) if $\dot{c}$ is time-like (causal). This extends naturally to continuous curves. A hypersurface $M \subset \bar{M}$ is called acausal if no causal curve meets $M$ more than once, and is called space-like if the normal of $M$ is time-like.

Given an acausal closed subset $S \subset \bar{M}$, the domain of dependence $D(S)$ is the set of points $p \in \bar{M}$ such that any inextendible causal curve containing $p$ must intersect $S$. If $S \subset \bar{M}$ is a space-like hypersurface and $D(S)=\bar{M}, S$ is called a Cauchy surface in $\bar{M}$. If $\bar{M}$ has a Cauchy surface $\bar{M}$ is called globally hyperbolic. A globally hyperbolic spacetime has a global time function, i.e. a function $t$ on $\bar{M}$ so that $\bar{\nabla} t$ is time-like, with the level sets of $t$ being Cauchy surfaces. In particular, $\bar{M} \cong M \times \mathbb{R}$ where $M$ is any Cauchy surface.

$\bar{M}$ is called time oriented if there is a global time-like vector field on $\bar{M}$. A globally hyperbolic spacetime is time oriented by the above, and hence it makes sense to talk about the future and past domains of dependence $D^{+}(M)$ and $D^{-}(M)$ of a Cauchy surface $M$. In a time oriented space time, the chronological future $I^{+}(S)$ of $S \subset \bar{M}$ is the set of all points reached by future directed time-like curves, of nonzero length, starting on $S$. The timelike past $I^{-}(S)$ is defined analogously. The causal future and past $J^{+}(S)$ and $J^{-}(S)$ is defined analogously to $I^{ \pm}(S)$ with causal curve replacing time-like curve (the causal curve is allowed to be trivial).

The variational problem for geodesics (w.r.t. Lorentzian length) is well behaved precisely when $(\bar{M}, \bar{g})$ is globally hyperbolic. Let $C(p, q)$ be the set of continuous causal curves between $p, q \in \bar{M}$. Then $C(p, q)$ is compact w.r.t. uniform convergence for all $p, q \in \bar{M}$, if $(\bar{M}, \bar{g})$ is globally hyperbolic. This makes global hyperbolicity a natural assumption in Lorentzian geometry, just as completeness is a natural assumption in Riemannian geometry.

Global hyperbolicity may also be characterized by strong causality and compactness of $C(p, q)$ for all $p, q \in \bar{M}$, or compactness of $J^{+}(p) \cap J^{-}(q)$ for all $p, q \in \bar{M}$, see [165, Chapter 8] for details.

Given a spacetime $(\bar{M}, \bar{g})$ and a space-like hypersurface $M \subset \bar{M}$, the future Cauchy horizon $H^{+}(M)$ is given by $H^{+}(M)=\overline{D^{+}(M)} \backslash I^{-}\left[D^{+}(M)\right]$, with the past Cauchy horizon $H^{-}(M)$ defined analogously. The set $H(M)=$ $H^{+}(M) \cup H^{-}(M)$ is called the Cauchy horizon of $M$. It can be proved that $H(M)=\partial D(M)$, the boundary of $D(M)$.

\section{REFERENCES}

1. Miguel Alcubierre, Appearance of coordinate shocks in hyperbolic formalisms of general relativity, Phys. Rev. D (3) 55 (1997), no. 10, 5981-5991.

2. Miguel Alcubierre and Joan Massó, Pathologies of hyperbolic gauges in general relativity and other field theories, Phys. Rev. D (3) 57 (1998), no. 8, R4511-R4515. 
3. Lars Andersson, Constant mean curvature foliations of flat space-times, Comm. Anal. Geom. 10 (2002), no. 5, 1125-1150.

4. Lars Andersson and Piotr T. Chruściel, Hyperboloidal Cauchy data for vacuum Einstein equations and obstructions to smoothness of null infinity, Phys. Rev. Lett. 70 (1993), no. 19, 2829-2832.

5. __ On "hyperboloidal" Cauchy data for vacuum Einstein equations and obstructions to smoothness of scri, Comm. Math. Phys. 161 (1994), no. 3, 533-568.

6. Lars Andersson, Piotr T. Chruściel, and Helmut Friedrich, On the regularity of solutions to the Yamabe equation and the existence of smooth hyperboloidal initial data for Einstein's field equations, Comm. Math. Phys. 149 (1992), no. 3, 587-612.

7. Lars Andersson, Gregory J. Galloway, and Ralph Howard, A strong maximum principle for weak solutions of quasi-linear elliptic equations with applications to Lorentzian and Riemannian geometry, Comm. Pure Appl. Math. 51 (1998), no. 6, 581-624.

8. Lars Andersson and Mirta S. Iriondo, Existence of constant mean curvature hypersurfaces in asymptotically flat spacetimes, Ann. Global Anal. Geom. 17 (1999), no. 6, 503-538.

9. Lars Andersson and Vincent Moncrief, Future complete vacuum spacetimes, gr$\mathrm{qc} / 0303045$, in this volume.

10. , Elliptic-hyperbolic systems and the Einstein equations, Ann. Henri Poincaré 4 (2003), no. 1, 1-34.

11. Lars Andersson, Vincent Moncrief, and Anthony J. Tromba, On the global evolution problem in $2+1$ gravity, J. Geom. Phys. 23 (1997), no. 3-4, 191-205.

12. Lars Andersson and Alan D. Rendall, Quiescent cosmological singularities, Comm. Math. Phys. 218 (2001), no. 3, 479-511.

13. Lars Andersson, Henk van Elst, and Claes Uggla, Gowdy phenomenology in scalefree variables, Classical Quantum Gravity 21 (2004), S29-S57, Spacetime Safari: Essays in Honor of Vincent Moncrief on the Classical Physics of Strong Gravitational Fields, special issue of Classical and Quantum Gravity, eds. J. Isenberg and B. Berger.

14. Håkan Andréasson, Alan D. Rendall, and Marsha Weaver, Existence of CMC and constant areal time foliations in $T^{2}$ symmetric spacetimes with Vlasov matter, Comm. Partial Differential Equations 29 (2004), no. 1-2, 237-262.

15. Abhay Ashtekar, Jiří Bičák, and Bernd G. Schmidt, Asymptotic structure of symmetry-reduced general relativity, Phys. Rev. D (3) 55 (1997), no. 2, 669-686.

16. , Behavior of Einstein-Rosen waves at null infinity, Phys. Rev. D (3) 55 (1997), no. 2, 687-694.

17. Abhay Ashtekar and Madhavan Varadarajan, Striking property of the gravitational Hamiltonian, Phys. Rev. D (3) 50 (1994), no. 8, 4944-4956.

18. Hajer Bahouri and Jean-Yves Chemin, Équations d'ondes quasi-linéaires et estimations de Strichartz, C. R. Acad. Sci. Paris Sér. I Math. 327 (1998), no. 9, 803-806.

19. M. Salah Baouendi and Charles Goulaouic, Remarks on the abstract form of nonlinear Cauchy-Kovalevsky theorems, Comm. Partial Differential Equations 2 (1977), no. 11, 1151-1162.

20. John D. Barrow, Gregory J. Galloway, and Frank J. Tipler, The closed-universe recollapse conjecture, Mon. Not. R. Astron. Soc. 223 (1986), 835-844.

21. Robert Bartnik, Existence of maximal surfaces in asymptotically flat spacetimes, Comm. Math. Phys. 94 (1984), no. 2, 155-175.

22. ㄴ Regularity of variational maximal surfaces, Acta Math. 161 (1988), no. 3-4, $145-181$.

23. Remarks on cosmological spacetimes and constant mean curvature surfaces, Comm. Math. Phys. 117 (1988), no. 4, 615-624.

24. John K. Beem, Paul E. Ehrlich, and Kevin L. Easley, Global Lorentzian geometry, second ed., Marcel Dekker Inc., New York, 1996. 
25. R. Beig and N. Ó Murchadha, Late time behaviour of the maximal slicing of the Schwarzschild black hole, Phys. Rev. D (3) 57 (1998), 4728-4737, gr-qc/9706046.

26. B. K. Berger and D. Garfinkle, Phenomenology of the Gowdy universe on $T^{3} \times R$, Phys. Rev. D57 (1998), 4767-4777, gr-qc/9710102.

27. Beverly K. Berger, Piotr T. Chruściel, James Isenberg, and Vincent Moncrief, Global foliations of vacuum spacetimes with $T^{2}$ isometry, Ann. Physics 260 (1997), no. 1, $117-148$.

28. Beverly K. Berger, David Garfinkle, James Isenberg, Vincent Moncrief, and Marsha Weaver, The singularity in generic gravitational collapse is spacelike, local and oscillatory, Modern Phys. Lett. A 13 (1998), no. 19, 1565-1573.

29. Beverly K. Berger, David Garfinkle, and Vincent Moncrief, Comment on "The Gowdy $T^{3}$ cosmologies revisited", gr-qc/9708050.

30. Beverly K. Berger, David Garfinkle, and Eugene Strasser, New algorithm for Mixmaster dynamics, Classical Quantum Gravity 14 (1997), no. 2, L29-L36.

31. Beverly K. Berger, James Isenberg, and Marsha Weaver, Oscillatory approach to the singularity in vacuum spacetimes with $T^{2}$ isometry, Phys. Rev. D (3) 64 (2001), no. 8, 084006, 20.

32. Beverly K. Berger and Vincent Moncrief, Evidence for an oscillatory singularity in generic $\mathrm{U}(1)$ symmetric cosmologies on $T^{3} \times \mathbf{R}$, Phys. Rev. D (3) 58 (1998), 064023, gr-qc/9804085.

33. __ Numerical evidence that the singularity in polarized $\mathrm{U}(1)$ symmetric cosmologies on $T^{3} \times \mathbf{R}$ is velocity dominated, Phys. Rev. D (3) 57 (1998), no. 12, 7235-7240, gr-qc/9801078.

34. Luc Blanchet and Thibault Damour, Hereditary effects in gravitational radiation, Phys. Rev. D (3) 46 (1992), no. 10, 4304-4319.

35. Dieter Brill and Frank Flaherty, Isolated maximal surfaces in spacetime, Comm. Math. Phys. 50 (1976), no. 2, 157-165.

36. Gregory A. Burnett and Alan D. Rendall, Existence of maximal hypersurfaces in some spherically symmetric spacetimes, Classical Quantum Gravity 13 (1996), no. 1, $111-123$.

37. John Cameron and Vincent Moncrief, The reduction of Einstein's vacuum equations on spacetimes with spacelike U(1)-isometry groups, Mathematical aspects of classical field theory (Seattle, WA, 1991), Amer. Math. Soc., Providence, RI, 1992, pp. 143169.

38. Myeongju Chae and Piotr T. Chruściel, On the dynamics of Gowdy space-times, Comm. Pure Appl. Math. 57 (2004), no. 8, 1015-1074.

39. Yvonne Choquet-Bruhat, Future complete Einsteinian space times with U(1) symmetry, the unpolarized case, article in this volume, gr-qc/0305060.

40. Yvonne Choquet-Bruhat and Robert Geroch, Global aspects of the Cauchy problem in general relativity, Comm. Math. Phys. 14 (1969), 329-335.

41. Yvonne Choquet-Bruhat and Vincent Moncrief, Existence theorem for solutions of Einstein's equations with 1 parameter spacelike isometry groups, Quantization, nonlinear partial differential equations, and operator algebra (Cambridge, MA, 1994), Proc. Symp. Pure Math., vol. 59, Amer. Math. Soc., Providence, RI, 1996, pp. 67-80.

42. _ Future global in time Einsteinian spacetimes with $\mathrm{U}(1)$ isometry group, Ann. Henri Poincaré 2 (2001), no. 6, 1007-1064.

43. Yvonne Choquet-Bruhat and Tommaso Ruggeri, Hyperbolicity of the $3+1$ system of Einstein equations, Comm. Math. Phys. 89 (1983), no. 2, 269-275.

44. Yvonne Choquet-Bruhat and James W. York, Mixed elliptic and hyperbolic systems for the Einstein equations, gr-qc/9601030.

45. Demetrios Christodoulou, The instability of naked singularities in the gravitational collapse of a scalar field, Ann. of Math. (2) 149 (1999), no. 1, 183-217. 
46. Demetrios Christodoulou and Sergiu Klainerman, The global nonlinear stability of the Minkowski space, Princeton University Press, Princeton, NJ, 1993.

47. Piotr T. Chruściel, On space-times with $\mathrm{U}(1) \times \mathrm{U}(1)$ symmetric compact Cauchy surfaces, Ann. Physics 202 (1990), no. 1, 100-150.

48. _ On uniqueness in the large of solutions of Einstein's equations ("strong cosmic censorship"), Proceedings of the Centre for Mathematics and its Applications, Australian National University, vol. 27, Australian National University Centre for Mathematics and its Applications, Canberra, 1991.

49. _ On uniqueness in the large of solutions of Einstein's equations ("strong cosmic censorship"), Mathematical aspects of classical field theory (Seattle, WA, 1991), Amer. Math. Soc., Providence, RI, 1992, pp. 235-273.

50. 10 (1993), no. 10, 2091-2101, ITP Preprint NSF-ITP-93-44.

51. Piotr T. Chruściel and Erwann Delay, Existence of non-trivial, vacuum, asymptotically simple spacetimes, Classical Quantum Gravity 19 (2002), no. 9, L71-L79, Erratum, Classical Quantum Gravity 19 (2002), no. 12, 3389.

52. Piotr T. Chruściel and Gregory J. Galloway, Horizons non-differentiable on a dense set, Comm. Math. Phys. 193 (1998), no. 2, 449-470.

53. Piotr T. Chruściel and James Isenberg, Nonisometric vacuum extensions of vacuum maximal globally hyperbolic spacetimes, Phys. Rev. D (3) 48 (1993), no. 4, 1616-1628.

54. Piotr T. Chruściel, James Isenberg, and Vincent Moncrief, Strong cosmic censorship in polarised Gowdy spacetimes, Classical Quantum Gravity 7 (1990), no. 10, 16711680 .

55. Piotr T. Chruściel and Alan D. Rendall, Strong cosmic censorship in vacuum spacetimes with compact, locally homogeneous Cauchy surfaces, Ann. Physics 242 (1995), no. 2, 349-385.

56. Piotr T. Chruściel and Jalal Shatah, Global existence of solutions of the YangMills equations on globally hyperbolic four-dimensional Lorentzian manifolds, Asian J. Math. 1 (1997), no. 3, 530-548.

57. Neil J. Cornish and Janna Levin, The mixmaster univese: A chaotic Farey tale, Phys. Rev. D (3) 55 (1997), 7489-7510, gr-qc/9612066.

58. Justin Corvino, Scalar curvature deformation and a gluing construction for the Einstein constraint equations, Comm. Math. Phys. 214 (2000), no. 1, 137-189.

59. _ Scalar curvature deformation and a gluing construction for the Einstein constraint equations, Comm. Math. Phys. 214 (2000), no. 1, 137-189.

60. Sergio Dain and Helmut Friedrich, Asymptotically flat initial data with prescribed regularity at infinity, Comm. Math. Phys. 222 (2001), no. 3, 569-609.

61. T. Damour, M. Henneaux, A. D. Rendall, and M. Weaver, Kasner-like behaviour for subcritical Einstein-matter systems, Ann. Henri Poincaré 3 (2002), no. 6, 1049-1111.

62. Douglas M. Eardley, Edison Liang, and Rainer Kurt Sachs, Velocity dominated singularities in irrotational dust cosmologies, J. Math. Phys. 13 (1972), 99-107.

63. Douglas M. Eardley and Vincent Moncrief, The global existence of Yang- MillsHiggs fields in 4-dimensional Minkowski space. I. Local existence and smoothness properties, Comm. Math. Phys. 83 (1982), no. 2, 171-191.

64. _ The global existence of Yang-Mills-Higgs fields in 4-dimensional Minkowski space. II. Completion of proof, Comm. Math. Phys. 83 (1982), no. 2, 193-212.

65. Klaus Ecker and Gerhard Huisken, Parabolic methods for the construction of spacelike slices of prescribed mean curvature in cosmological spacetimes, Comm. Math. Phys. 135 (1991), no. 3, 595-613.

66. G. F. R. Ellis and M. A. H. MacCallum, A class of homogeneous cosmological models, Comm. Math. Phys. 12 (1969), 108-141.

67. Arthur Fischer and Vincent Moncrief, Hamiltonian reduction of Einstein's equations of general relativity, Nuclear Physics B (Proc. Suppl) 57 (1997), 142-161. 
68. Arthur E. Fischer, Jerrold E. Marsden, and Vincent Moncrief, The structure of the space of solutions of Einstein's equations. I. One Killing field, Ann. Inst. H. Poincaré Sect. A (N.S.) 33 (1980), no. 2, 147-194.

69. Helmut Friedrich, On the existence of n-geodesically complete or future complete solutions of Einstein's field equations with smooth asymptotic structure, Comm. Math. Phys. 107 (1986), no. 4, 587-609.

70. - On the global existence and the asymptotic behavior of solutions to the Einstein-Maxwell-Yang-Mills equations, J. Differential Geom. 34 (1991), no. 2, 275345.

71. Hyperbolic reductions for Einstein's equations, Classical Quantum Gravity 13 (1996), no. 6, 1451-1469.

72. Helmut Friedrich, István Rácz, and Robert M. Wald, On the rigidity theorem for spacetimes with a stationary event horizon or a compact Cauchy horizon, grqc/9811021, 1998.

73. Simonetta Frittelli and Oscar A. Reula, First-order symmetric-hyperbolic Einstein equations with arbitrary fixed gauge, Phys. Rev. Lett 76 (1996), 4667-4670, grqc/9605005.

74. Yoshihisa Fujiwara, Hideki Ishihara, and Hideo Kodama, Comments on closed Bianchi models, Classical Quantum Gravity 10 (1993), no. 5, 859-867, grqc/9301019.

75. Gregory J. Galloway, The Lorentzian splitting theorem without the completeness assumption, J. Differential Geom. 29 (1989), no. 2, 373-387.

76. Some rigidity results for spatially closed spacetimes, Mathematics of gravitation, Part I (Warsaw, 1996), Polish Acad. Sci., Warsaw, 1997, pp. 21-34.

77. David Garfinkle and Marsha Weaver, High velocity spikes in Gowdy spacetimes, Phys. Rev. D (3) 67 (2003), no. 12, 124009, 5.

78. Claus Gerhardt, H-surfaces in Lorentzian manifolds, Comm. Math. Phys. 89 (1983), no. $4,523-553$.

79. __ Hypersurfaces of prescribed mean curvature in Lorentzian manifolds, Math. Z. 235 (2000), no. 1, 83-97.

80. Robert H. Gowdy, Gravitational waves in closed universes, Phys. Rev. Lett. 27 (1971), 826-829.

81. _ Vacuum spacetimes with two-parameter spacelike isometry groups and compact invariant hypersurfaces: topologies and boundary conditions, Ann. Physics 83 (1974), 203-241.

82. Boro Grubišić and Vincent Moncrief, Asymptotic behavior of the $T^{3} \times \mathbf{R}$ Gowdy space-times, Phys. Rev. D (3) 47 (1993), no. 6, 2371-2382, gr-qc/9209006.

83. - Mixmaster spacetime, Geroch's transformation, and constants of motion, Phys. Rev. D (3) 49 (1994), no. 6, 2792-2800, gr-qc/9309007.

84. S. W. Hawking and G. F. R. Ellis, The large scale structure of space-time, Cambridge University Press, London, 1973, Cambridge Monographs on Mathematical Physics, No. 1.

85. Oliver Henkel, Global prescribed mean curvature foliations in cosmological spacetimes. I, II, J. Math. Phys. 43 (2002), no. 5, 2439-2465, 2466-2485.

86. Simon D. Hern and John M. Stewart, The Gowdy $T^{3}$ cosmologies revisited, Classical Quantum Gravity 15 (1998), no. 6, 1581-1593.

87. Conrad G Hewitt, Joshua T Horwood, and John Wainwright, Asymptotic dynamics of the exceptional Bianchi cosmologies, gr-qc/0211071, 2002.

88. David Hobill, Adrian Burd, and Alan Coley (eds.), Deterministic chaos in general relativity, New York, Plenum Press, 1994.

89. Lars Hörmander, Lectures on nonlinear hyperbolic differential equations, SpringerVerlag, Berlin, 1997. 
90. Thomas J. R. Hughes, Tosio Kato, and Jerrold E. Marsden, Well-posed quasi-linear second-order hyperbolic systems with applications to nonlinear elastodynamics and general relativity, Arch. Rational Mech. Anal. 63 (1976), no. 3, 273-294 (1977).

91. Gerhard Huisken and Tom Ilmanen, The Riemannian Penrose inequality, Internat. Math. Res. Notices 1997, no. 20, 1045-1058.

92. - The inverse mean curvature flow and the Riemannian Penrose inequality, J. Differential Geom. 59 (2001), no. 3, 353-437.

93. Mirta S. Iriondo, Enzo O. Leguizamón, and Oscar A. Reula, Einstein's equations in Ashtekar's variables constitute a symmetric hyperbolic system, Phys. Rev. Lett 79 (1997), 4732-4735, gr-qc/9710004.

94. On the dynamics of Einstein's equations in the Ashtekar formulation, Adv. Theor. Math. Phys. 2 (1998), no. 5, 1075-1103.

95. James Isenberg, Progress on strong cosmic censorship, Mathematical aspects of classical field theory (Seattle, WA, 1991), Amer. Math. Soc., Providence, RI, 1992, pp. 403-418.

96. James Isenberg and Satyanad Kichenassamy, Asymptotic behavior in polarized $T^{2}$ symmetric vacuum space-times, J. Math. Phys. 40 (1999), no. 1, 340-352.

97. James Isenberg, Rafe Mazzeo, and Daniel Pollack, Gluing and wormholes for the Einstein constraint equations, Comm. Math. Phys. 231 (2002), no. 3, 529-568.

98. - On the topology of vacuum spacetimes, Ann. Henri Poincaré 4 (2003), no. 2, 369-383, gr-qc/0206034.

99. James Isenberg and Vincent Moncrief, The existence of constant mean curvature foliations of Gowdy 3-torus spacetimes, Comm. Math. Phys. 86 (1982), no. 4, 485493

100. _ Symmetries of cosmological Cauchy horizons with exceptional orbits, J. Math. Phys. 26 (1985), no. 5, 1024-1027.

101. Asymptotic behavior of the gravitational field and the nature of singularities in Gowdy spacetimes, Ann. Physics 199 (1990), no. 1, 84-122.

102. __ Asymptotic behaviour in polarized and half-polarized U(1) symmetric vacuum spacetimes, Classical Quantum Gravity 19 (2002), no. 21, 5361-5386.

103. James Isenberg and Alan D. Rendall, Cosmological spacetimes not covered by a constant mean curvature slicing, Classical Quantum Gravity 15 (1998), no. 11, 36793688.

104. Akihiro Ishibashi, Tatsuhiko Koike, Masaru Siino, and Sadayoshi Kojima, Compact hyperbolic universe and singularities, Phys. Rev. D (3) 54 (1996), no. 12, 7303-7310, gr-qc/9605041.

105. Ronald Kantowski and Rainer Kurt Sachs, Some spatially homogeneous anisotropic relativistic cosmological models, J. Mathematical Phys. 7 (1966), 443-446.

106. Michael Kapovich, Deformations of representations of discrete subgroups of $\mathrm{SO}(3,1)$, Math. Ann. 299 (1994), no. 2, 341-354.

107. Satyanad Kichenassamy and Alan D. Rendall, Analytic description of singularities in Gowdy spacetimes, Classical Quantum Gravity 15 (1998), no. 5, 1339-1355, preprint at http://www.aei-potsdam.mpg.de.

108. S. Klainerman, On the regularity of classical field theories in Minkowski space-time $\mathbf{R}^{3+1}$, Nonlinear partial differential equations in geometry and physics (Knoxville, TN, 1995), Birkhäuser, Basel, 1997, pp. 29-69.

109. Sergiu Klainerman and Matei Machedon, Finite energy solutions of the Yang-Mills equations in $\mathbf{R}^{3+1}$, Ann. of Math. (2) 142 (1995), no. 1, 39-119.

110. Sergiu Klainerman and Francesco Nicolò, On local and global aspects of the Cauchy problem in general relativity, Classical Quantum Gravity 16 (1999), R73-R157.

111. The evolution problem in general relativity, Progress in Mathematical Physics, vol. 25, Birkhäuser Boston Inc., Boston, MA, 2003. 
112. _ Peeling properties of asymptotically flat solutions to the Einstein vacuum equations, Classical and Quantum Gravity 20 (2003), no. 14, 3215-3257.

113. Sergiu Klainerman and Igor Rodnianski, The causal structure of microlocalized Einstein metrics, math.AP/0109174, 2001.

114. _ Rough solution for the Einstein vacuum equations, math.AP/0109173, 2001.

115. Sergiu Klainerman and Igor Rodnianski, Ricci defects of microlocalized Einstein metrics, J. Hyperbolic Differ. Equ. 1 (2004), no. 1, 85-113.

116. Sergiu Klainerman and Sigmund Selberg, Remark on the optimal regularity for equations of wave maps type, Comm. Partial Differential Equations 22 (1997), no. 5-6, 901-918.

117. Hideo Kodama, Canonical structure of locally homogeneous systems on compact closed 3-manifolds of types $E^{3}$, Nil and Sol, Progr. Theoret. Phys. 99 (1998), no. 2, $173-236$.

118. Tatsuhiko Koike, Masayuki Tanimoto, and Akio Hosoya, Compact homogeneous universes, J. Math. Phys. 35 (1994), no. 9, 4855-4888.

119. Joachim Krieger, Global regularity of wave maps from $\mathbf{r}^{2+1}$ to $\mathbf{h}^{2}$, preprint.

120. E. M. Lifshitz and I. M. Khalatnikov, Investigations in relativistic cosmology, Adv. in Phys. 12 (1963), 185-249.

121. Xue-Feng Lin and Robert M. Wald, Proof of the closed-universe-recollapse conjecture for diagonal Bianchi type-IX cosmologies, Phys. Rev. D (3) 40 (1989), no. 10, 32803286.

122. Hans Lindblad, Counterexamples to local existence for quasilinear wave equations, Math. Res. Lett. 5 (1998), no. 5, 605-622.

123. Hans Lindblad and Igor Rodnianski, The weak null condition for Einstein's equations, C. R. Math. Acad. Sci. Paris 336 (2003), no. 11, 901-906.

124. Jerrold E. Marsden and Frank J. Tipler, Maximal hypersurfaces and foliations of constant mean curvature in general relativity, Phys. Rep. 66 (1980), no. 3, 109-139.

125. Vincent Moncrief, Global properties of Gowdy spacetimes with $T^{3} \times \mathbf{R}$ topology, Ann. Physics 132 (1981), no. 1, 87-107.

126. _ Reduction of Einstein's equations for vacuum space-times with spacelike $\mathrm{U}(1)$ isometry groups, Ann. Physics 167 (1986), no. 1, 118-142.

127. L Reduction of Einstein's equations for cosmological spacetimes with spacelike U(1)-isometry groups, Physique quantique et géométrie (Paris, 1986), Hermann, Paris, 1988, pp. 105-117.

128. _ Reduction of the Einstein-Maxwell and Einstein-Maxwell-Higgs equations for cosmological spacetimes with spacelike U(1) isometry groups, Classical Quantum Gravity 7 (1990), no. 3, 329-352.

129. Vincent Moncrief and Douglas M. Eardley, The global existence problem and cosmic censorship in general relativity, Gen. Relativity Gravitation 13 (1981), no. 9, 887892.

130. Vincent Moncrief and James Isenberg, Symmetries of cosmological Cauchy horizons, Comm. Math. Phys. 89 (1983), no. 3, 387-413.

131. Peter Orlik and Frank Raymond, On 3-manifolds with local $\mathrm{SO}(2)$ action, Quart. J. Math. Oxford Ser. (2) 20 (1969), 143-160.

132. R. Penrose, Techniques of differential topology in relativity, SIAM, Philadelphia, PA., 1972.

133. R. Penrose, Some unsolved problems in classical general relativity, Seminar on Differential Geometry, Princeton Univ. Press, Princeton, N.J., 1982, pp. 631-668.

134. Alan D. Rendall, Global properties of locally spatially homogeneous cosmological models with matter, Math. Proc. Cambridge Philos. Soc. 118 (1995), no. 3, 511-526.

135. Constant mean curvature foliations in cosmological space-times, Helv. Phys. Acta 69 (1996), no. 4, 490-500, Journées Relativistes 96, Part II (Ascona, 1996), gr-qc/9606049. 
136. _ Existence of constant mean curvature foliations in spacetimes with twodimensional local symmetry, Comm. Math. Phys. 189 (1997), no. 1, 145-164.

137. Global dynamics of the mixmaster model, Classical Quantum Gravity 14 (1997), no. 8, 2341-2356.

138. _ Fuchsian analysis of singularities in Gowdy spacetimes beyond analyticity, Classical Quantum Gravity 17 (2000), no. 16, 3305-3316.

139. Theorems on existence and global dynamics for the Einstein equations, Living Rev. Relativ. 5 (2002), 2002-6, 62 pp. (electronic).

140. Alan D. Rendall and Marsha Weaver, Manufacture of Gowdy spacetimes with spikes, Classical Quantum Gravity 18 (2001), no. 15, 2959-2975.

141. Hans Ringström, Curvature blow up in Bianchi VIII and IX vacuum spacetimes, Classical Quantum Gravity 17 (2000), no. 4, 713-731.

142. _ The Bianchi IX attractor, Ann. Henri Poincaré 2 (2001), no. 3, 405-500.

143. _ Asymptotic expansions close to the singularity in Gowdy spacetimes, Classical Quantum Gravity 21 (2004), S305-S322.

144. _ On Gowdy vacuum spacetimes, Math. Proc. Cambridge Philos. Soc. 136 (2004), no. 2, 485-512.

145. Peter Scott, The geometries of 3-manifolds, Bull. London Math. Soc. 15 (1983), no. 5, 401-487.

146. Jalal Shatah, The Cauchy problem for harmonic maps on Minkowski space, Proceedings of the International Congress of Mathematicians, Vol. 1, 2 (Zürich, 1994) (Basel), Birkhäuser, 1995, pp. 1126-1132.

147. Stephen T. C. Siklos, Occurrence of whimper singularities, Comm. Math. Phys. 58 (1978), no. 3, 255-272.

148. Larry Smarr and Jr. York, James W., Kinematical conditions in the construction of spacetime, Phys. Rev. D (3) 17 (1978), no. 10, 2529-2551.

149. _ Radiation gauge in general relativity, Phys. Rev. D (3) 17 (1978), no. 8, 1945-1956.

150. Hart F. Smith and Christopher D. Sogge, On Strichartz and eigenfunction estimates for low regularity metrics, Math. Res. Lett. 1 (1994), no. 6, 729-737.

151. Hart F. Smith and Daniel Tataru, Sharp local well-posedness results for the nonlinear wave equation, http://www.math.berkeley.edu/ tataru/nlw.html, 2001.

152. Chrisopher D. Sogge, Lectures on nonlinear wave equations, Monographs in Analysis, vol. II, International Press, Cambridge, MA, 1995.

153. Christopher D. Sogge, On local existence for nonlinear wave equations satisfying variable coefficient null conditions, Comm. Partial Differential Equations 18 (1993), no. $11,1795-1821$.

154. _ Fourier integral operators and nonlinear wave equations, Mathematics of gravitation, Part I (Warsaw, 1996), Polish Acad. Sci., Warsaw, 1997, pp. 91-108.

155. Masayuki Tanimoto, New varieties of Gowdy space-times, J. Math. Phys. 39 (1998), no. 9, 4891-4898.

156. Terence Tao, Global regularity of wave maps. II. Small energy in two dimensions, Comm. Math. Phys. 224 (2001), no. 2, 443-544.

157. Daniel Tataru, Nonlinear wave equations, Proceedings of the ICM, Beijing 2002, vol. 3, 2003, pp. 209-220, math.AP/0304397.

158. _ Rough solutions for the wave-maps equation, http://math.berkeley.edu/ tataru/nlw.html, 2003.

159. Daniel Tataru, The wave maps equation, Bull. Amer. Math. Soc. (N.S.) 41 (2004), no. 2, 185-204 (electronic).

160. William P. Thurston, Three-dimensional geometry and topology. Vol. 1, Princeton University Press, Princeton, NJ, 1997, Edited by Silvio Levy.

161. Claes Uggla, Henk van Elst, John Wainwright, and George F. R. Ellis, The past attractor in inhomogenous cosmology, Phys. Rev. D 68 (2003), 103502. 
162. Juan Antonio Valiente Kroon, A new class of obstructions to the smoothness of null infinity, Comm. Math. Phys. 244 (2004), no. 1, 133-156.

163. John Wainwright and George F. R. Ellis (eds.), Dynamical systems in cosmology, Cambridge University Press, Cambridge, 1997, Papers from the workshop held in Cape Town, June 27-July 2, 1994.

164. John Wainwright and Lucas Hsu, A dynamical systems approach to Bianchi cosmologies: orthogonal models of class A, Classical Quantum Gravity 6 (1989), no. 10, 1409-1431.

165. Robert M. Wald, General relativity, University of Chicago Press, Chicago, Ill., 1984.

166. _ Gravitational collapse and cosmic censorship, Black holes, gravitational radiation and the universe, Fund. Theories Phys., vol. 100, Kluwer Acad. Publ., Dordrecht, 1999, pp. 69-85.

167. Marsha Weaver, Dynamics of magnetic Bianchi $\mathrm{VI}_{0}$ cosmologies, Classical Quantum Gravity 17 (2000), no. 2, 421-434, gr-qc/9909043.

168. Marsha Weaver, James Isenberg, and Beverly K. Berger, Mixmaster behavior in inhomogeneous cosmological spacetimes, Phys. Rev. Lett 80 (1998), 2984-2987, grqc/9712055.

Department of Mathematics, University of Miami, Coral Gables, FL 33124, USA

E-mail address: larsa@math.miami.edu 